\title{
A method for the calculation of inter-element stresses in 3D
}

\author{
Daniela Ciancio ${ }^{\mathrm{a}}$, Ignacio Carol $^{\mathrm{b}}$, Massimo Cuomo $^{\mathrm{c}}$ \\ "School of Civil and Resoturce Engineering. UWA (Univ. of Westem Australia), 6009 Perth, Australia \\ ${ }^{b}$ ETSECCPB (School of Civil Engineering), UAC (Tech. Univ. of Catalonia), 08034 Barcelona, Spain \\ ${ }^{\mathrm{C} D I C A}$ (Department of Civil and Environmental Engineeringd. University of Catania, 95125 Catania, Italy
}

\begin{abstract}
A B S T R A C T
In a previous paper, the authors have proposed a local post processing method out of the standard results of 2D FE displacement calculations, for the evaluation of the "inter element" forces and stress tractions transmitted across mesh lines. The method is based on a double minimization of the differences between the inter element tractions and the projections on the same lines of the (also initially unknown) nodal stress tensor. In the present paper, the method is extended to 3D. Inter element lines become surfaces, and overall geometric and equilibrium conditions turn out significantly more complex. In spite of that, a formulation based on the same principles is developed and demonstrated with basic numerical appli cations. The method is always applicable for linear elements but it shows some peculiarities for the cor ner nodes of some quadratic elements.
\end{abstract}

\section{Introduction}

In a previous paper [1], the authors have proposed a procedure for the evaluation of inter element forces and stress tractions in 2D, which computationally takes the form of a local post processing calculation at each node of a standard FE mesh (displacement for mulation). The motivation for this development was the evaluation of the stress tractions transmitted between adjacent elements in the context of a new general procedure for the representation of cracking and fracture along mesh lines based on the cohesive crack model [2], of which another ingredient is described in [3]. The pro posed method represents the first step of this general procedure that is the evaluation of the triggering conditions for the nucleation of a crack in an intact material.

Most existing procedures that represent cracking along mesh lines are based on the systematic insertion of double node inter face elements from the beginning of the calculations [4 8] with the subsequent increase of the number of nodes and computa tional requirements. Alternatively, some authors have proposed to duplicate nodes and insert interfaces only as the cracking con ditions are reached $\left[\begin{array}{ll}9 & 12\end{array}\right]$. But this requires the evaluation of in ter element forces in the original mesh without interfaces, something that in 2D is a trivial operation only at mid side nodes of quadratic elements. For this reason, in some of those refer ences, six node triangular elements are used and cracking condi tions are evaluated initially at mid side nodes (whose opening represents unique crack path), and at corner nodes that can potentially open up along multiple fracture paths. To overcome this limitation, in a previous paper [1] the authors have proposed a 2D formulation in which inter element forces and stresses may be evaluated at the "corner" nodes of the mesh for both quadratic and linear elements, therefore making it possible to check crack ing conditions at any node.

In the present paper, the 2D formulation proposed by the authors [1] is extended and demonstrated also in 3D. In addition to the already mentioned applications in fracture mechanics, the proposed method can have further areas of practice. Other applica tions could be, for instance, the extraction of inter element forces for practical design of a retaining wall or tunnel lining, including then rock mechanics and geotechnical engineering.

After this introduction, the paper is structured as follows: in Section 2 a definition of inter element forces is presented. In Sec tion 3, the original 2D procedure is briefly summarized. In Section 4 the formulation for the 3D case is presented: Section 4.1 includes the basic count of unknowns and equations at a generic inner cor ner node, the notation for all static variables, the equilibrium rela tions between them, the objective function and the minimizations; Section 4.2 treats the special case of inter element forces and stres ses at the mid edge nodes of quadratic elements; Section 4.3 re peats the procedure for the special cases of corner nodes on a boundary surface. Section 5 includes an additional related discus sion on the concept of contributing area. Section 6 includes some simple examples of application of the formulation. Finally, some concluding remarks of this work are presented in Section 7. 


\section{Nodal forces of zero-thickness interface elements}

The zero thickness finite interface elements provide a good start ing point for introducing the new procedure proposed in this paper. Interfaces are always located in between two standard continuum elements, with which they share their element sides or faces. As re sult of the analysis one obtains stress tractions along the interface contact, here denoted as $\mathbf{t}$, which by definition are at the same time the stress tractions acting on the contiguous element side faces. Interface elements are defined in such way [13] that the interpola tion functions coincide with those of the adjacent continuum ele ment specified for the corresponding element side face. Let us consider for simplicity the 2D case, and denote with $s$ the curvilinear coordinate system measured along the interface domain $e$. The shape functions on that domain are $\mathbf{N}(s)$, with as many components as nodes on the inter element face. Standard application of the Prin ciple of Virtual Work (PVW) leads to the classical expression:

$\mathbf{f}_{s} \quad \int_{e} \mathbf{N}^{t} \mathbf{t} d s$

in which in this case the vector $\mathbf{f}_{s}$ gathers the collection of the inter element forces $\mathbf{r}_{i}(i \quad 1, N)$ transmitted through the $N$ nodes across the inter element contact surface. In the context of a cohesive crack analysis along mesh lines, the importance of the evaluation of such inter element forces and (especially) tractions is evident, since the cracking criterion takes in general the form of a plastic surface in terms of the stress traction components. It seems also reasonable that (in analogy to standard element forces obtained via PVW or similar principles) those forces $\mathbf{r}_{i}$ satisfy the equilibrium conditions at the nodes in the strong sense.

\section{Brief outline of the procedure in 2D}

The original problem under study can be easily introduced in 2D by a simple count of unknowns and equilibrium equations at a gen eric nodal point where $N$ element tips and $N$ mesh (or inter element) lines concur (see Fig. 1).

The number of unknowns $N$ corresponds to the inter element forces denoted as $\mathbf{r}^{(k)}(k \quad 1, N)$. The vectorial equilibrium equa tions for each of the $N$ element tips concurrent at the node may be expressed as follows: $\left\{\begin{array}{lll}\mathbf{f}^{(1)} & \mathbf{r}^{(N)}+\mathbf{r}^{(1)} & \mathbf{0} \\ \mathbf{f}^{(2)} & \mathbf{r}^{(1)}+\mathbf{r}^{(2)} & \mathbf{0} \\ \mathbf{f}^{(3)} & \mathbf{r}^{(2)}+\mathbf{r}^{(3)} & \mathbf{0} \\ \vdots & & \\ \mathbf{f}^{(N)} & \left.\mathbf{r}^{(N} 1\right)+\mathbf{r}^{(N)} & \mathbf{0}\end{array}\right.$

in which $\mathbf{f}^{(k)}$ are the (known) element nodal forces transmitted by ele ment $k$ to the node, which may be obtained a posteriori of the FE anal ysis using the classical weak equilibrium equation for the element:

$\mathbf{f}_{e} \quad \int_{V_{e}} \mathbf{B}^{T} \boldsymbol{\sigma} d V_{e}$,

where $V_{e}$ is the element volume, $\boldsymbol{\sigma}$ the stress field in the element and $\mathbf{B}$ the matrix containing the derivatives of the element shape functions.

However, only $N \quad 1$ of the vector equations in (2) are really independent as reflected by the fact that the sum of all of them leads to an identity, i.e., $\sum_{i}^{N}{ }_{1} \mathbf{f}^{(i)} \quad \mathbf{0}$. This may be interpreted as that any of the unknowns, for instance the force $\overline{\mathbf{r}} \quad \mathbf{r}^{(1)}$, may be ta ken as arbitrary variables, which means that the system would ex hibit infinite solutions, one for each given values of $\overline{\mathbf{r}}$.

By considering the global to local $\left((\mathbf{x}, \mathbf{y})\right.$ to $\left(\mathbf{n}^{(k)}, \mathbf{t}^{(k)}\right)$ as shown in Fig. 1) rotation matrix $\mathbf{Q}^{(k)}$ for the inter element line $k$ and the con tributing area $\Omega^{(k)}$ of the corresponding corner node along that line, the nodal inter element forces may be converted into nodal inter element stress tractions $\mathbf{t}^{(k)} \quad\left(\sigma^{(k)}, \tau^{(k)}\right)^{T} \quad \mathbf{Q}^{(k)} \mathbf{r}^{(k)} / \Omega^{(k)}$. Please note that the contributing area $\Omega$ in this case is the product of the con tributing length along the mesh line and the unit thickness perpen dicular to the analysed 2D plane.

The (initially unknown) stress tensor at the node $\mathbf{T}$ is intro duced, and an objective function is defined equal to the sum of the squares of the differences between the inter element tractions and the projections of $\mathbf{T}$ on the same directions:

$\Phi \quad \sum_{k 1}^{N}\left[\left(\sigma^{(k)}(\overline{\mathbf{r}}) \quad n^{(k)} \mathbf{T} n^{(k)}\right)^{2}+\left(\tau^{(k)}(\overline{\mathbf{r}}) \quad t^{(k)} \mathbf{T} n^{(k)}\right)^{2}\right]$.

For the first minimization, the arbitrary force and therefore the overall set of inter element tractions are assumed to be known, and the objective function is minimized with respect to each of the independent components $T_{i j}$ (three in 2D). This leads to an
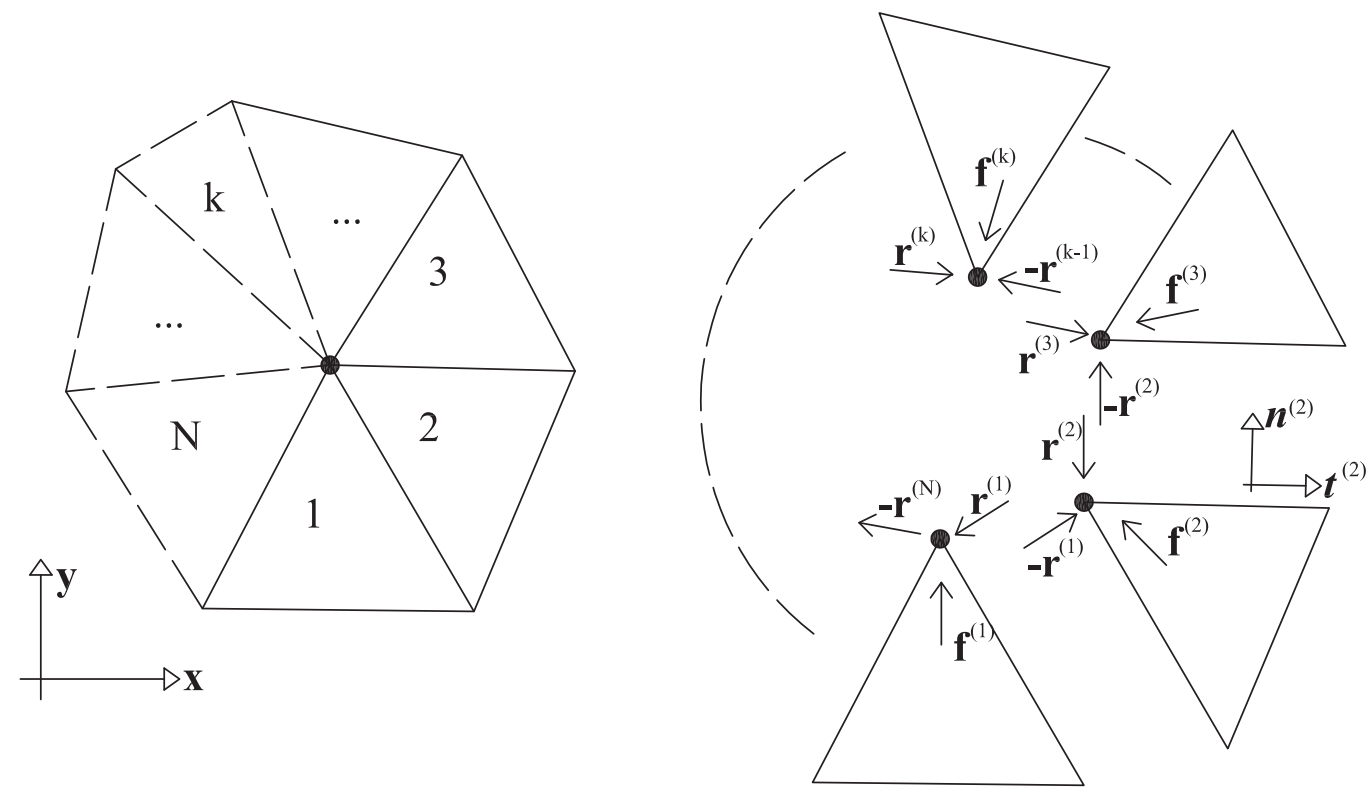

Fig. 1. Element $\mathbf{f}^{(k)}$ and inter-element $\mathbf{r}^{(k)}$ nodal forces along the mesh lines concurring at a generic interior nodal point. 
explicit expression of the stress tensor $\mathbf{T}$ in terms of all inter ele ment tractions. It is important to observe that, if the element stress tractions were exact, the obtained stress tensor would also be exact.

The previous solution is then backsubstituted into the objec tive function, which now becomes a function of the arbitrary var iable $\overline{\mathbf{r}}$ exclusively, or in other words of its components $\bar{r}_{j}(j=1,2)$. This function is minimized a second time with respect to $\bar{r}_{j}$, lead ing to a 2 linear equation system. In this linear system, the coef ficient matrix depends only on geometric factors (i.e., contributing areas and orientations of mesh lines concurring at the node) while the right hand side vector contains the continu ous element nodal forces.

The numerical solution of this two by two algebraic linear sys tem produces the values of the arbitrary force $\overline{\mathbf{r}}$ and, by backsubsti tution, the inter element forces, stress tractions, and stress tensor at the node. More details can be found in [1].

It is important to emphasise that the inter element traction vec tors obtained by the proposed minimization when multiplied by their relative contributing areas become inter element forces that are in equilibrium with the nodal forces of the continuous elements. This is a significant attribute of the recovered stresses. The same can not be said for the traction vectors obtained by, for instance, an aver age recovery procedure which is less onerous from the computational point of view but does not return nodal inter ele ment forces equilibrated with the continuous nodal forces.

For the 2D formulation presented in [1], a comparison between the proposed minimization procedure and an average recovery method was presented (please, refer to Fig. 18 and 19 of [1]). The study showed that the proposed method gives better results than the average procedure, especially for boundary nodes. This conclu sion can be easily extended to 3D.

One last observation on the $2 \mathrm{D}$ formulation regards the calcula tion of inter element stresses around a notch. In 2D this situation is analogous to the case of a boundary node in which, for $N$ elements concurrent at the node, $N \quad 1$ are the independent equilibrium equations and $N \quad 1$ are the unknown inter element forces. The proposed minimization method would therefore be not required since the inter element forces and stresses could be recovered by simple application of the equilibrium equations alone.

\section{Procedure in 3D}

For a generic 3D FE simulation, the nodes of an inter element surface can be distinguished into corner, mid edge and center face nodes, as shown in Fig. 2 (the latter 2 are only in the case of qua dratic elements). The calculation of the inter element nodal forces at interior center face nodes and at boundary mid edge nodes is trivial because (similarly to the mid edge nodes in 2D) it is possible by equilibrium equations alone. In this section the evaluation pro cedure for inter element forces and stress tractions is presented for the case of interior corner nodes, interior mid edge nodes and boundary corner nodes.

\subsection{Formulation for interior corner nodes}

\subsubsection{Count of unknowns}

In contrast to the 2D case, in which the count of unknowns and arbitrary variables was simple, in 3D it depends on the type of ele ments (prisms, hexahedra, etc.) and their arrangement around the analysed node. In particular, denoting by:

- $N$ the total number of elements around the node, and

- $p_{i}$ the number of faces for each element $i(i=1, N)$ at the node,

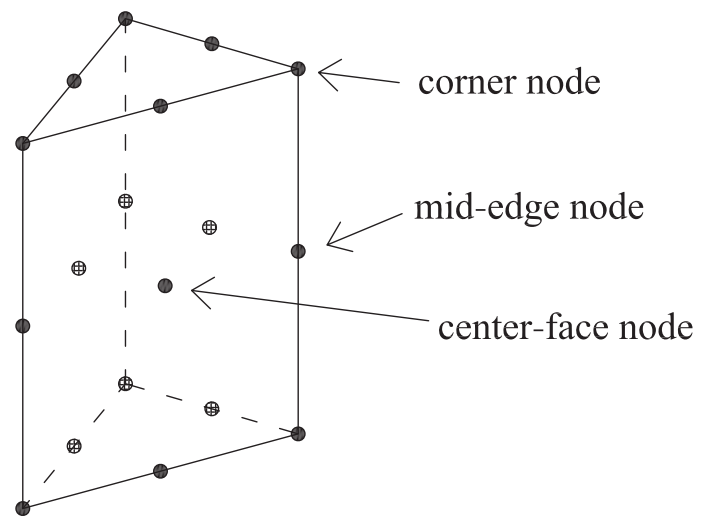

Fig. 2. Characterization of corner nodes, mid-edge nodes and center-face nodes.

one can define:

- the total number of scalar unknowns: $3 v \quad 3 \sum_{i}^{N}{ }_{1} p_{i} / 2$

- the number of scalar independent equilibrium equations: $3\left(\begin{array}{ll}N & 1\end{array}\right)$

- the number of scalar arbitrary variables: $\begin{array}{lllll}3 s & 3 v & 3\left(\begin{array}{ll}N & 1\end{array}\right)\end{array}$ $3\left(\sum_{i 1}^{N} p_{i} / 2 \quad N+1\right)$

The above formulas can be verified in the following examples. Fig. 3 represents six tetrahedra around a node for which $N \quad 6$ and $p_{i} \quad 3$ (for $i$ 1,6). This leads to:

- total number of unknowns: $3 v \quad 3 \sum_{i i_{1}}^{6}\left(p_{i}\right) / 2 \quad 3 * 9 \quad 27$

- number of independent equilibrium equations: $3\left(\begin{array}{ll}N & 1\end{array}\right) \quad 3 * 5 \quad 15$

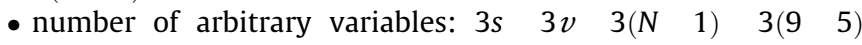
12

The example in Fig. 4 shows four tetrahedra and three pyramids around a node. In this case $N \quad 7, p_{1} \quad p_{2} \quad p_{3} \quad p_{4} 3$ and $p_{5} \quad p_{6} \quad p_{7} \quad 4$, which leads to:

- total number of unknowns: $3 v \quad 3 \sum_{i{ }_{1}}^{7}\left(p_{i}\right) / 2 \quad 3 * 12 \quad 36$

- number of independent equilibrium equations: $3\left(\begin{array}{ll}N & 1\end{array}\right)$ $3 * 6 \quad 18$

- number of arbitrary variables: $\left.\begin{array}{lllll}3 s & 3 v & 3 & (N & 1\end{array}\right)$ $3(12 \quad 6) \quad 18$
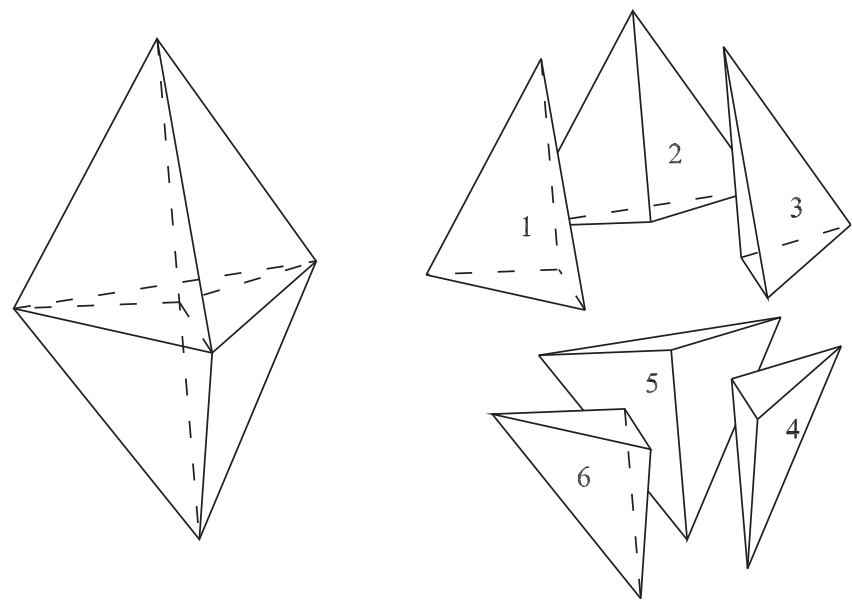

Fig. 3. Example of 6 tetrahedra around an interior corner node. 

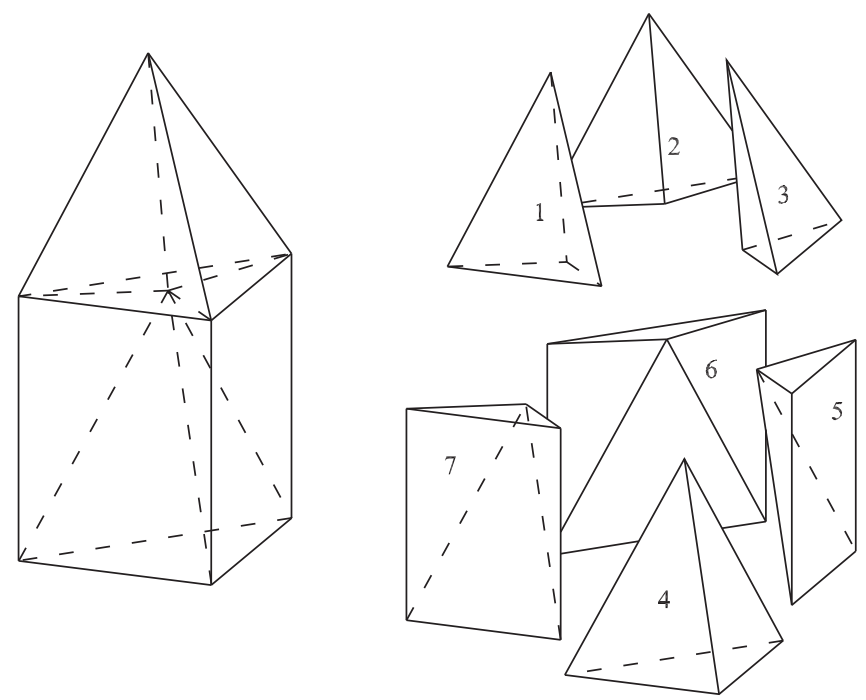

Fig. 4. Example of four tetrahedra and three pyramids around a node.

\subsubsection{Formulation}

Fig. 5 shows the element nodal forces (on the left side) and the inter element forces (on the right side) around a node. The con cepts and equations reported in the following refer to this particu lar example (six tetrahedra) but may be also extended to any number $N$ of solid elements concurrent at the node.

The inter element stress traction evaluation method is a post processing procedure, and this implies that the FE mesh has been solved previously under certain boundary conditions, that all the nodal displacements have been computed and from them the strains and stresses at the Gauss points, and finally the force vectors for each element have been recovered using the weak equilibrium equation in (3). Let us call $\mathbf{T}$ the (initially unknown) stress tensor at the node. Similarly to the approach used in the 2D formulation, on the basis of the well known "engineering" or Voigt notation [14], $\mathbf{T}$ is presented here as a column vector which only includes the six independent components in the global reference system $(x, y, z)$. The vectorial notation adopted here leads to manageable expressions for the proposed procedure. An analogous approach in tensor notation is also possible. However, it leads to a complex system [19] that makes impractical to obtain simple closed form solutions and it is not presented in this paper.

For the example in Fig. 5, the set of equilibrium equations at the tip of each element around the interior corner node (similar to Eq. (2) in 2D), can be written in matrix form as:

$$
\left[\begin{array}{cccccc|ccc}
\mathbf{I} & \mathbf{0} & \mathbf{I} & \mathbf{0} & \mathbf{0} & \mathbf{0} & \mathbf{0} & -\mathbf{I} & \mathbf{0} \\
-\mathbf{I} & \mathbf{I} & \mathbf{0} & \mathbf{0} & \mathbf{0} & \mathbf{0} & \mathbf{I} & \mathbf{0} & \mathbf{0} \\
\mathbf{0} & -\mathbf{I} & \mathbf{0} & \mathbf{0} & \mathbf{0} & \mathbf{I} & \mathbf{0} & \mathbf{I} & \mathbf{0} \\
\mathbf{0} & \mathbf{0} & -\mathbf{I} & \mathbf{I} & \mathbf{0} & \mathbf{0} & \mathbf{0} & \mathbf{0} & -\mathbf{I} \\
\mathbf{0} & \mathbf{0} & \mathbf{0} & -\mathbf{I} & \mathbf{I} & \mathbf{0} & -\mathbf{I} & \mathbf{0} & \mathbf{0}
\end{array}\right]\left[\begin{array}{c}
\mathbf{r}^{(1)} \\
\mathbf{r}^{(2)} \\
\mathbf{r}^{(3)} \\
\mathbf{r}^{(4)} \\
\mathbf{r}^{(5)} \\
\hline \mathbf{r}^{(6)} \\
\mathbf{r}^{(7)} \\
\mathbf{r}^{(8)} \\
\mathbf{r}^{(9)}
\end{array}\right]=\left[\begin{array}{c}
\mathbf{f}^{(1)} \\
\mathbf{f}^{(2)} \\
\mathbf{f}^{(3)} \\
\mathbf{f}^{(4)} \\
\mathbf{f}^{(5)}
\end{array}\right]
$$

The partition lines in the previous equation are meant to separate the total number of inter element forces $\left(\begin{array}{ll}v & 9\end{array}\right)$ in two subsets: a first group including a number of forces equal to the number of lin ear independent vectorial equations $N \quad 1 \quad\left(\begin{array}{ll}6 & 1\end{array}\right) \quad 5$, and a sec ond group with the remaining $v \quad\left(\begin{array}{llll}N & 1\end{array}\right) \quad 9 \quad 5 \quad 4$ forces, which
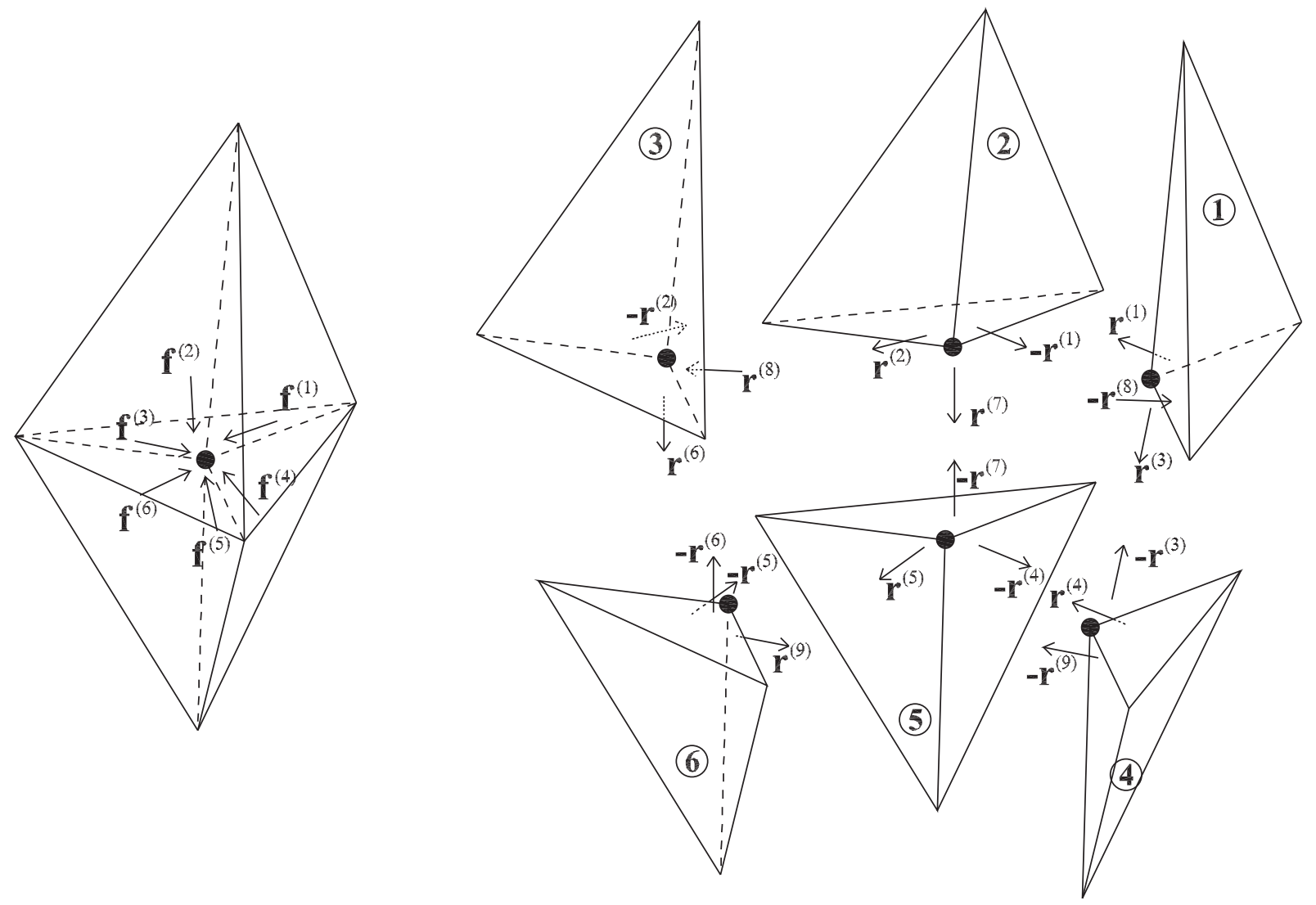

Fig. 5. Element nodal forces (left) and inter-element nodal forces (right) for a set of six tetrahedral elements surrounding a node. 

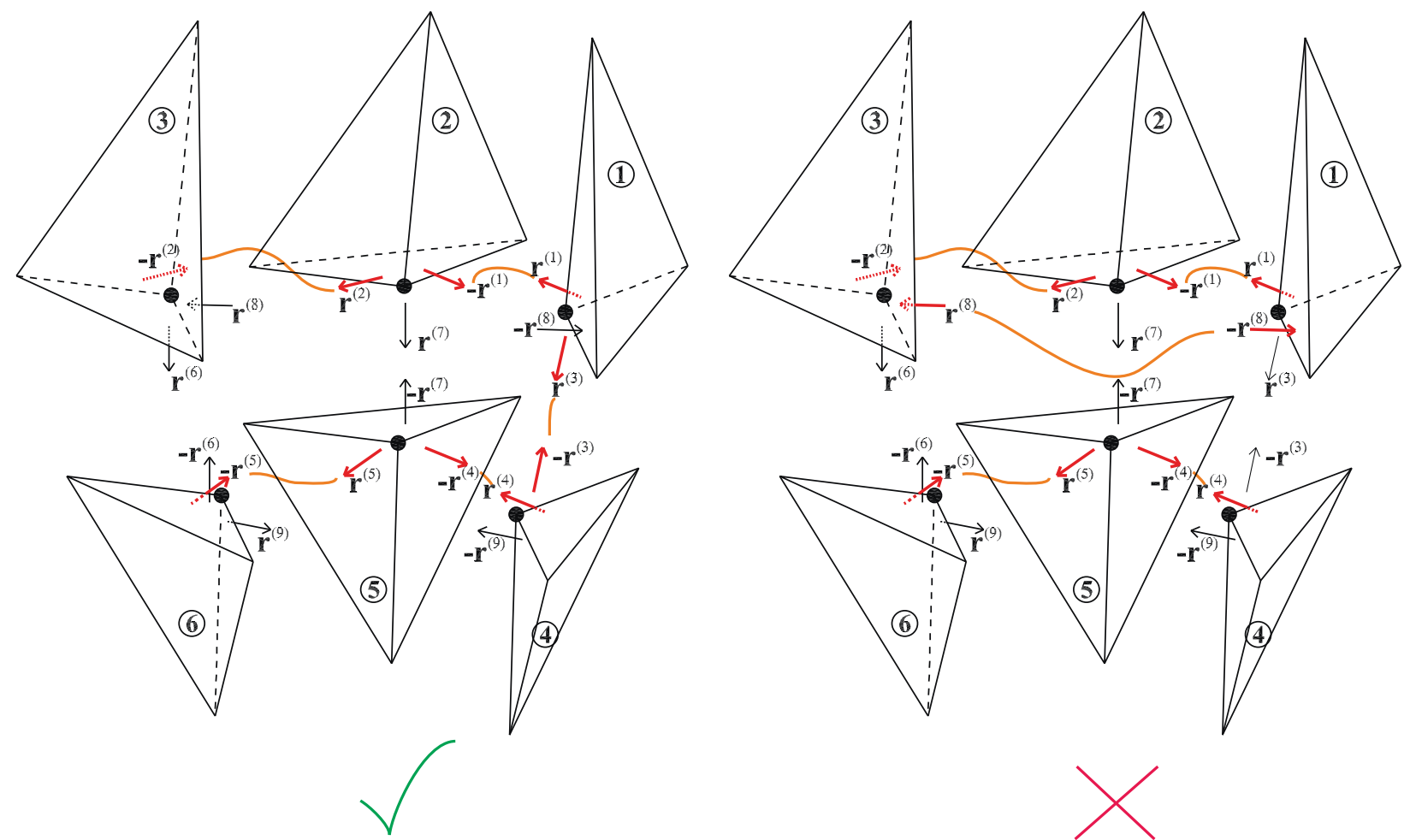

Fig. 6. Choice of independent inter-element nodal forces $\mathbf{r}^{(k)}$ : correct choice (left) and incorrect choice (right).

may be considered as arbitrary forces. Note that, all the components of the previous expression are themselves $3 \times 3$ matrices (for the case of $\mathbf{I}$ and $\mathbf{0}$ ) or 3 component vectors (for $\mathbf{f}^{(i)}$ and $\mathbf{r}^{(i)}$ ).

Expression (5) may be rewritten in compact form as:

$$
\mathbf{H r} \quad \mathbf{f} \text { or }\left[\begin{array}{ll}
\mathbf{H}_{0} & \mathbf{H}_{1}
\end{array}\right]\left[\begin{array}{l}
\mathbf{r}_{0} \\
\mathbf{r}_{1}
\end{array}\right] \quad[\mathbf{f}]
$$

where $\mathbf{H}_{0}$ and $\mathbf{H}_{1}$ are matrices with dimensions $\left[3\left(\begin{array}{ll}N & 1\end{array}\right) \times 3\left(\begin{array}{ll}N & 1\end{array}\right)\right]$ and $\left[3\left(\begin{array}{ll}N & 1\end{array}\right) \times 3 s\right]$ respectively, $\mathbf{r}_{0}$ and $\mathbf{r}_{1}$ are vectors with $3(N 1)$ and $3 s$ components, and $\mathbf{f}$ is another vector with $3\left(\begin{array}{l}N \\ 1)\end{array}\right)$ components. In Eq. (6) one can easily isolate $\mathbf{r}_{0}$ in terms of the arbitrary forces $\mathbf{r}_{1}$ :

$$
\begin{array}{rll}
\mathbf{r}_{0} & \mathbf{H}_{0}{ }^{1}(\mathbf{f} & \left.\mathbf{H}_{1} \mathbf{r}_{1}\right) .
\end{array}
$$

Note that, while in 2D any inter element force $\mathbf{r}^{(i)}$ could be selected as arbitrary force $\overline{\mathbf{r}}$ (as explained in Section 3), in 3D this choice is not arbitrary at the risk of obtaining a singular matrix $\mathbf{H}_{0}$. This can be avoided if the following two conditions are satisfied:

- the $N 1$ forces in $\mathbf{r}_{0}$ continuously "connect" all the continuum elements around the node, and

- there are no more than $p_{i} \quad 1$ forces selected for each element $i$ ( $p_{i}=$ number of faces of element $i$ at the node).

This is illustrated with the examples of Fig. 6, which depicts a cor rect choice on the left image, and an incorrect choice on the right one. Note that the above conditions can be easily implemented in the form of a simple automatic algorithm.

In a general case other than the example in Fig. 5, one can as sume that $\mathbf{r}$ is a $[3 v]$ vector containing all the inter element forces which, by taking into account (7) can be written as:

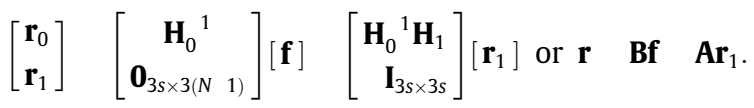

As it was done in $2 \mathrm{D}$, the following step in the procedure is to con vert the inter element forces $\mathbf{r}^{(i)}$ into inter element stress tractions $\mathbf{t}^{(i)}$ which are needed in the objective function for the minimization procedure. This is easily done by means of the contributing area $\Omega^{(i)}$ (Fig. 7). Further details and fine points on the contributing area con cept are discussed in Section 5. Note that, in contrast to the 2D for mulation, no rotation matrix is considered here because the objective function will be expressed directly in terms of global cartesian components of the stress tractions (instead of the local normal and tangential components).

The force traction relation for an individual inter element sur face can therefore be expressed as:

$\mathbf{t}^{(i)} \frac{1}{\Omega^{(i)}} \mathbf{r}^{(i)}$

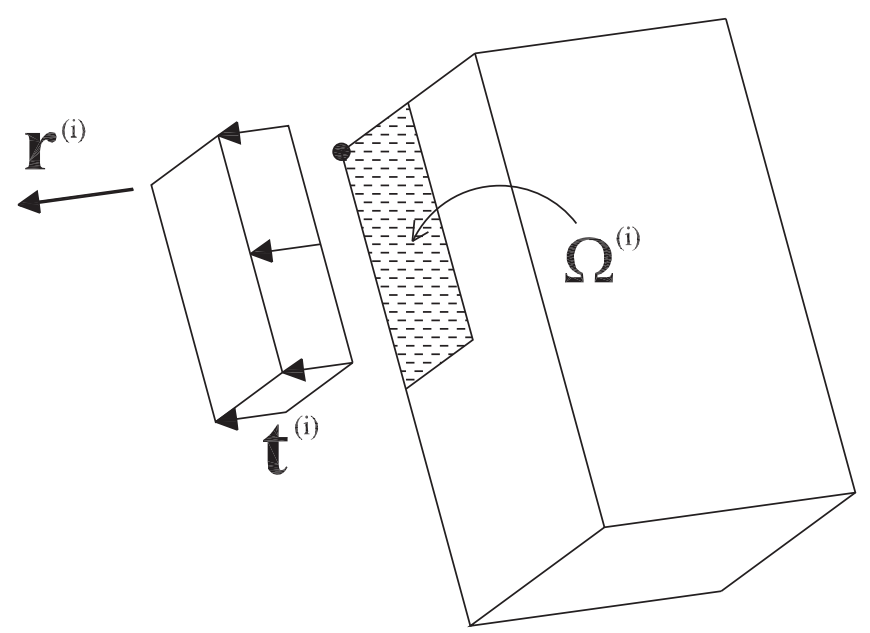

Fig. 7. Inter-element force $\mathbf{r}^{(i)}$, stress traction $\mathbf{t}^{(i)}$ and contributing area $\Omega^{(i)}$. 
The collection of all similar equations for each of the inter element faces surrounding a node leads to the expression:

$\mathbf{\Omega}{ }^{1} \mathbf{r}$

in which $\mathbf{s}$ is a vector containing all the $[3 v]$ components of the stress tractions, and $\mathbf{\Omega}$ is a $[3 v \times 3 v]$ diagonal matrix containing the contributing areas. By substituting (8) into (10) the inter ele ment tractions $\mathbf{s}$ in terms of the arbitrary forces $\mathbf{r}_{1}$ become:

S $\quad \Omega^{1}\left(\begin{array}{ll}\mathbf{B f} & \mathbf{A r} \\ 1\end{array}\right)$.

Similarly to the 2D case, the procedure now implies the minimiza tion of an objective function $\Phi$, representing the square of the sum of the differences between the projection of the (initially unknown) stress tensor $\mathbf{T}$ on the element faces, and the stress traction vectors $\mathbf{t}^{(i)}$ transmitted across these same faces. Each inter element face is characterized by a unit vector $\mathbf{n}^{(i)}$ normal to the face. Thus, the above mentioned difference between inter element stress traction and nodal stress projection can be expressed as:

$$
\left[\begin{array}{c}
t_{x}^{(i)} \\
t_{y}^{(i)} \\
t_{z}^{(i)}
\end{array}\right] \quad\left[\begin{array}{cccccc}
n_{x}^{(i)} & 0 & 0 & n_{y}^{(i)} & 0 & n_{z}^{(i)} \\
0 & n_{y}^{(i)} & 0 & n_{x}^{(i)} & n_{z}^{(i)} & 0 \\
0 & 0 & n_{z}^{(i)} & 0 & n_{y}^{(i)} & n_{x}^{(i)}
\end{array}\right]\left[\begin{array}{c}
\sigma_{x} \\
\sigma_{y} \\
\sigma_{z} \\
\tau_{x y} \\
\tau_{x z} \\
\tau_{y z}
\end{array}\right] \quad \mathbf{t}^{(i)} \quad \mathbf{P}^{(i)} \mathbf{T}
$$

The objective function that for the 2D case was formulated in terms of local (normal, tangential) components (Section 3 of [1]), in this case is directly expressed in terms of global $(x, y, z)$ components, yielding to:

$\Phi \quad \sum_{i=1}^{v}\left\|\mathbf{t}^{(i)} \quad \mathbf{P}^{(i)} \mathbf{T}\right\|^{2} \quad \operatorname{tr}\left[\left(\begin{array}{lll}\mathbf{S} & \mathbf{P T}\end{array}\right)\left(\begin{array}{ll}\mathbf{S} & \mathbf{P T}\end{array}\right)^{T}\right]$,

where matrix $\mathbf{P}$ with dimensions $[3 v \times 6]$ is the column collection of all submatrices $\mathbf{P}^{(i)}$.

The minimization of $\Phi$ with respect to the components of $\mathbf{T}$ leads in this case to the following linear equation:

$\frac{\partial \Phi}{\partial \mathbf{T}} \quad \mathbf{N T} \quad \mathbf{c} \quad 0$

where

\section{$\mathbf{N} \quad \mathbf{P}^{T} \mathbf{P}$ and $\mathbf{c} \quad \mathbf{P}^{T} \mathbf{S}$.}

Note that the result of the minimization is a simple linear matrix equation. This gives the following solution for the stress tensor in terms of the inter element tractions:

\section{T $\quad \mathbf{N}^{1} \mathbf{c}$}

The availability of the simple closed form solution (16) makes it possible to complete the formulation in a way similar to the 2D case (Section 2 of [1]). According to that, the expression of $\mathbf{T}$ just ob tained, together with those of $\mathbf{N}$ and $\mathbf{c}(15)$ may be backsubstituted into the objective function (13), leading to:

$\Phi \operatorname{tr}(\boldsymbol{\Pi} \mathbf{s})^{2}$,

where $\Pi$ is the following $[3 v \times 3 v]$ square symmetric matrix:

П $\quad \mathbf{I}_{3 v \times 3 v} \quad \mathbf{P}\left(\mathbf{P}^{T} \mathbf{P}\right){ }^{1} \mathbf{P}^{T}$.

The inter element tractions $\mathbf{s}$ in (17) may now be replaced by (11) to obtain a new expression of the objective function in terms of arbitrary inter element forces. The objective function $\Phi$ may then be minimized with respect to the arbitrary inter element forces $\mathbf{r}_{1}$. By doing that, and taking into account that $\Pi \Pi$ П, one obtains a linear equation system for $\mathbf{r}_{1}$ :

$\frac{\partial \Phi}{\partial \mathbf{r}_{1}} \quad \mathbf{K r}_{1} \quad \mathbf{L f} \quad \mathbf{0}$

in which matrices $\mathbf{K}[3 s \times 3 s]$ and $\mathbf{L}\left[3 s \times 3\left(\begin{array}{ll}N & 1\end{array}\right)\right]$ have expressions:

$\mathbf{K} \quad \mathbf{A}^{T} \boldsymbol{\Omega}{ }^{1} \Pi \Omega{ }^{1} \mathbf{A}$ and $\mathbf{L} \quad \mathbf{A}^{T} \boldsymbol{\Omega}{ }^{1} \Pi \Omega{ }^{1} \mathbf{B}$.

Eq. (19) may be solved by simple inversion of $\mathbf{K}$ to obtain the final expression:

$\mathbf{r}_{1} \quad \mathbf{K}^{1} \mathbf{L f}$.

Eq. (21) constitute the missing equations to solve the overall prob lem under study, that is, to obtain the nodal inter element stress tractions (and forces) at the interior node of a FE mesh representing an intact (uncracked) material. Once the system (21) is solved, one can obtain the inter element stress traction vectors $\mathbf{t}^{(i)}$ (which form vector s) using Eq. (11), and the equilibrated inter element forces from Eq. (8). Note that, rather than 2 different minimizations, the same Eqs. (21) and (16) could have been obtained by first substitut ing (8) into (10), then (10) into (13) and finally simultaneously min imizing $\Phi$ with respect to $\mathbf{T}$ and $\mathbf{r}_{1}$.

Note also that, similar to the 2D case, the nodal stress tensor $\mathbf{T}$ that can also be obtained using Eq. (16). However, the nodal stress tensor evaluation is not the main objective of the procedure, but only a by product of the calculation (which is primarily focused on the evaluation of equilibrated inter element stress tractions).

\subsection{Formulation for interior mid edge nodes}

\subsubsection{Count of unknowns}

In Fig. 8, point A represents a generic interior mid edge node, and $Y_{1}, Y_{2}, \ldots, Y_{N}$ represent the inter element surfaces of the $N$ ele ments sharing node $A$. The global reference system is $(x, y, z)$. The direction of the mesh line to which node A belongs is indicated as $\mathbf{m}$. The plane perpendicular to $\mathbf{m}$ is indicated as $\Gamma$. A cartesian local reference system on this plane is defined so that two direc tions $\left(x^{*}, y^{*}\right)$ are contained in $\Gamma$, and the third one $z^{*}$ is normal to the plane (or parallel to $\mathbf{m}$ ).

Looking at the right side of Fig. 8, it is easy to note that all the $\mathbf{n}^{(i)}$ normal vectors to the inter element surfaces $Y_{i}$ are contained in the plane $\Gamma$. The case of the mid edge node shows many similar ities with the case of a corner node in 2D (see Fig. 1). In fact if $N$ is the number of elements sharing node $\mathrm{A}$, then $N$ is also the number of inter element surfaces and the number of unknown inter ele ment forces, and $N \quad 1$ is the number of linear independent equi librium equations.

\subsubsection{Formulation}

A formulation similar to that of Section 4.1.2 is developed for interior mid edge nodes, for which one has $v \quad N$ and $s \quad 1$. The stress tensor is again expressed in vector (Voigt) notation. The equilibrium equations take an algebraic form similar to the 2D case (Eq. 2), although in this case each force variable is a 3 component vector. When writing only the first $N \quad 1$ equilibrium equations which are independent, the counterpart of Eq. (5) in the case, for instance, of $N=5$ becomes:

$$
\left[\begin{array}{ccccc}
\mathbf{I} & \mathbf{0} & \mathbf{0} & \mathbf{0} & \mathbf{I} \\
\mathbf{I} & \mathbf{I} & \mathbf{0} & \mathbf{0} & \mathbf{0} \\
\mathbf{0} & \mathbf{I} & \mathbf{I} & \mathbf{0} & \mathbf{0} \\
\mathbf{0} & \mathbf{0} & \mathbf{I} & \mathbf{I} & \mathbf{0}
\end{array}\right]\left[\begin{array}{l}
\mathbf{r}^{(1)} \\
\mathbf{r}^{(2)} \\
\mathbf{r}^{(3)} \\
\mathbf{r}^{(4)} \\
\mathbf{r}^{(5)}
\end{array}\right] \quad\left[\begin{array}{l}
\mathbf{f}^{(1)} \\
\mathbf{f}^{(2)} \\
\mathbf{f}^{(3)} \\
\mathbf{f}^{(4)}
\end{array}\right]
$$



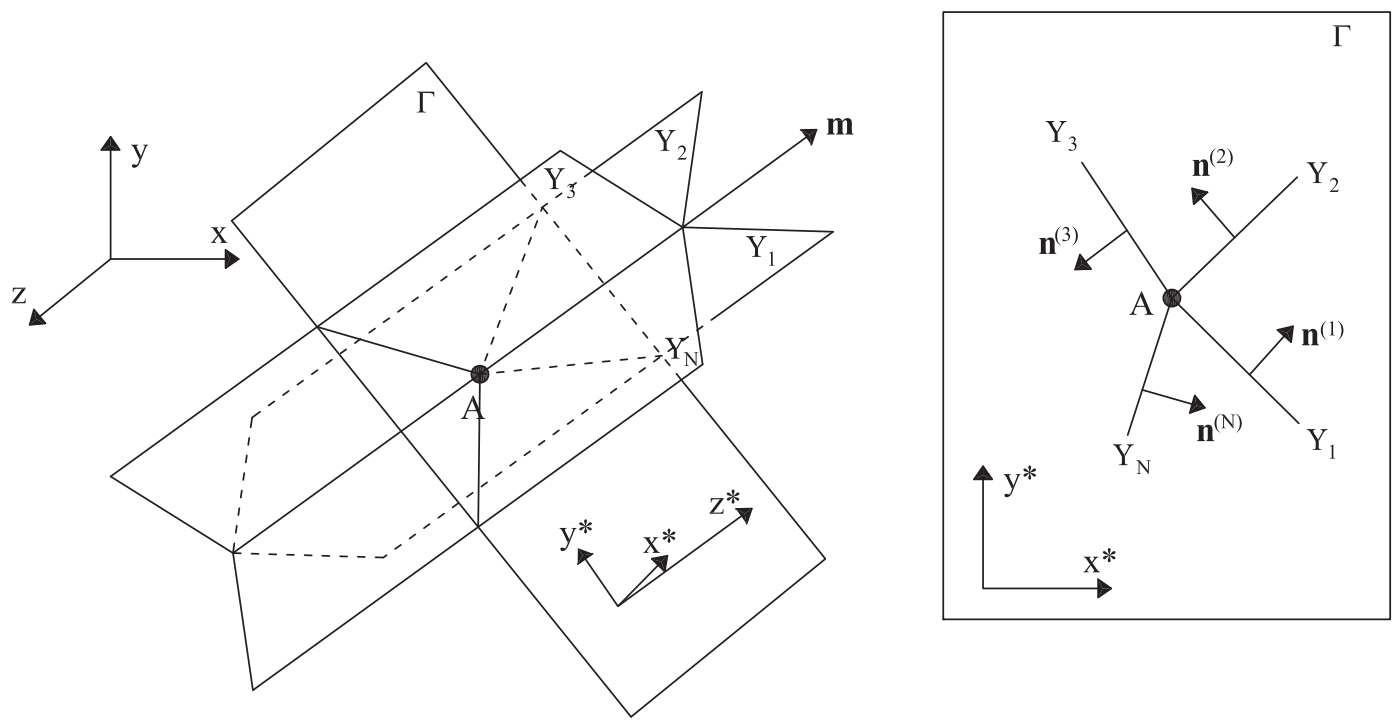

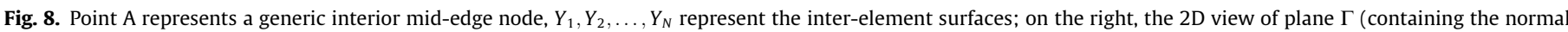
vectors $\mathbf{n}^{(i)}$ to $\left.Y_{i}, i=1, N\right)$ is offered.

which can be alternatively written in a more compact form as Eq. (6). Same arguments as in Section 4.1.2 lead to the expressions of $\mathbf{r}_{0}, \mathbf{r}$ and $\mathbf{s}$ in terms of $\mathbf{r}_{1}$, which are the same as in Section 4.1.2 (Eqs. (6) (11)).

However, from this point on, the formulation of the mid edge node differs from the one of the corner node. The difference be tween the nodal inter element traction in A along $\mathbf{n}^{(i)}$ and the pro jection of the stress tensor along the same direction can still be written as done in (12). However, in this case all normal vectors are contained in the same plane $\Gamma$, which would cause matrix $\mathbf{N}$ as defined in (15) to become singular.

In the local reference $\left(x^{*}, y^{*}, z^{*}\right)$, the generic normal vector be comes: $\mathbf{n}^{*(i)} \quad\left(n_{x_{*}}^{(i)}, n_{y^{*}}^{(i)}, 0\right)$. In this reference system, the counterpart of Eq. (12) is:

$$
\left[\begin{array}{c}
t_{x *}^{(i)} \\
t_{y *}^{(i)} \\
t_{z *}^{(i)}
\end{array}\right] \quad\left[\begin{array}{ccccc}
n_{x *}^{(i)} & 0 & n_{y^{*}}^{(i)} & 0 & 0 \\
0 & n_{y *}^{(i)} & n_{x_{*}}^{(i)} & 0 & 0 \\
0 & 0 & 0 & n_{x *}^{(i)} & n_{y *}^{(i)}
\end{array}\right]\left[\begin{array}{c}
\sigma_{x *} \\
\sigma_{y *} \\
\tau_{x * y *} \\
\tau_{x * z *} \\
\tau_{y * z *}
\end{array}\right] \quad \mathbf{t}^{(i)} \quad \mathbf{P}^{*(i)} \mathbf{T}^{*}
$$

Note that only five components of the stress tensor are contained in the new vector $\mathbf{T}^{*}$. The sixth component, representing the stress normal to the $\Gamma$ plane $\left(\sigma_{z}^{*}\right)$, does not take part in this formulation.

From this point onwards, the method for the mid edge node proceeds as for the corner node. An objective function (counterpart of (13)) is defined as:

$\Phi^{*} \quad \sum_{i=1}^{v}\left\|\mathbf{t}^{(i)} \quad \mathbf{P}^{*(i)} \mathbf{T}^{*}\right\|^{2} \quad \operatorname{tr}\left(\mathbf{S} \quad \mathbf{P}^{*} \mathbf{T}^{*}\right)^{2}$

The difference between (13) and (24) consists of the dimensions of matrices $\mathbf{P}$ and $\mathbf{P}^{*}$, being $[3 v \times 6]$ for the former and $[3 v \times 5]$ for the latter. The objective function $\Phi^{*}$ is minimised first with respect to $\mathbf{T}^{*}$ and then with respect to $\mathbf{r}_{1}$ obtaining the solution:

$$
\mathbf{r}_{1} \quad \mathbf{K}^{*}{ }^{1} \mathbf{L}^{*} \mathbf{f}
$$

with

$$
\begin{aligned}
& \mathbf{K}^{*} \quad \mathbf{A}^{T} \boldsymbol{\Omega}{ }^{1} \boldsymbol{\Pi}^{*} \boldsymbol{\Omega}{ }^{1} \mathbf{A}, \quad \mathbf{L}^{*} \quad \mathbf{A}^{T} \boldsymbol{\Omega}{ }^{1} \boldsymbol{\Pi}^{*} \boldsymbol{\Omega}{ }^{1} \mathbf{B} \text { and }
\end{aligned}
$$

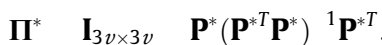

The solution in (25) makes it possible to obtain the values of the arbitrary nodal inter element force $\mathbf{r}_{1}$. All the nodal inter element stress tractions can then be obtained using (11), and the remaining nodal inter element forces evaluated from (7). Note, however, that in this case the proposed procedure does not lead to the recovery of the full stress tensor at the node, since $\mathbf{T}^{*}$ defined in (23) does not include the out of plane component $\sigma_{z}^{*}$.

\subsection{Formulation for boundary corner nodes}

\subsubsection{Count of unknowns}

For a boundary node to which $N$ continuum elements converge, the number of unknown vectorial inter element forces $s$ may be expressed as $s \quad v \quad\left(\begin{array}{ll}N & 1\end{array}\right)$, same formula as for standard inner nodes of the mesh (Section 4.1.1), except that in this case the total number of inter element forces is $v \quad \sum_{i}^{N}{ }_{1} p_{i}^{\prime} / 2$, being $p_{i}^{\prime}$ equal to the total number $p_{i}$ of faces meeting at the boundary node minus the faces on the boundary surface. In Figs. 9 and 10 two examples are presented which explain this count.

Fig. 9 shows four tetrahedra (i.e., $N \quad 4$ ) around node A. Each tetrahedron has 3 faces at A ( $p_{i} 3$ for $\left.i \quad 1,4\right)$, but only 2 of them are inter element faces $\left(p_{i}^{\prime} 2\right.$ for $\left.i \quad 1,4\right)$. For this example one has:

- total number of unknowns: $3 v \quad 3 \sum_{i 1}^{4}\left(p_{i}^{\prime}\right) / 2 \quad 3 * 4 \quad 12$

- number of independent equilibrium equations: $3\left(\begin{array}{ll}N & 1\end{array}\right) \quad 3 * 3 \quad 9$

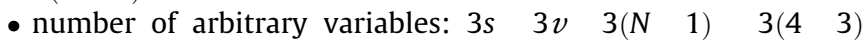
3

The example in Fig. 10 shows five pyramids around node A (i.e., in this case $N$ 5). Note that for pyramid 1, all the faces meeting at the boundary node $A$ are interior, and so $p_{1}^{\prime} \quad 4$. For pyramids 2, 3, 4 and 5 only three of the four faces meeting at the node are interior, so that $p_{2}^{\prime} \quad p_{3}^{\prime} \quad p_{4}^{\prime} \quad p_{5}^{\prime} \quad 3$. This leads to

- total number of unknowns: $3 v \quad 3 \sum_{i 1_{1}}^{5}\left(p_{i}^{\prime}\right) / 2 \quad 3 * 8 \quad 24$

- number of independent equilibrium equations: $3\left(\begin{array}{ll}N & 1\end{array}\right)$ $3 * 4 \quad 12$

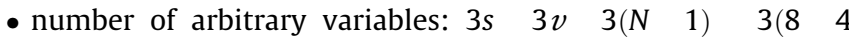
12 

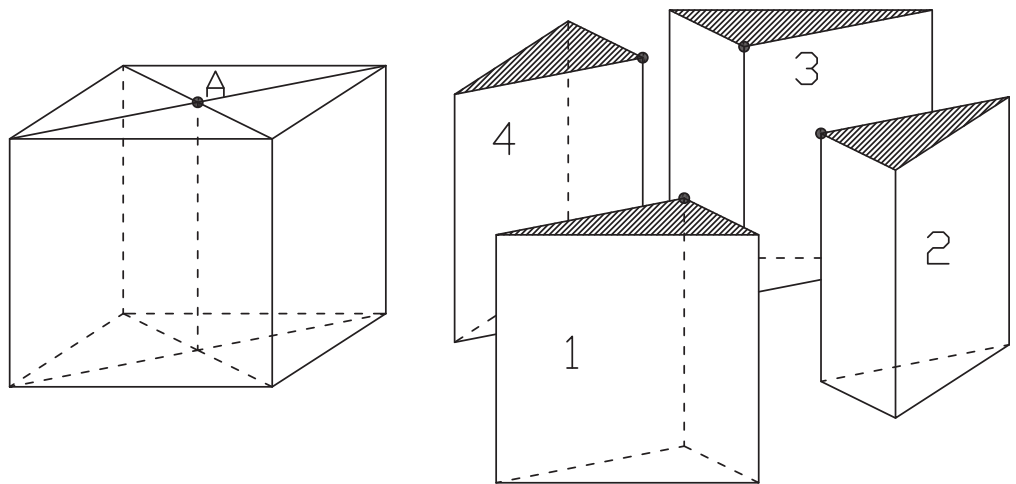

Fig. 9. Four triangular prisms meet at boundary node A; each element has one face on the boundary surface (shaded areas) and two inter-element faces.
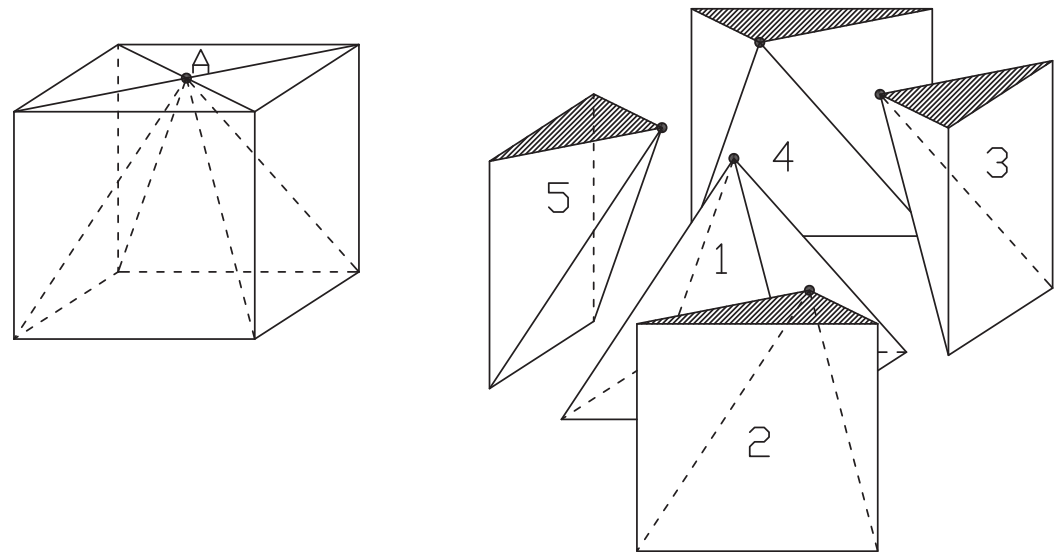

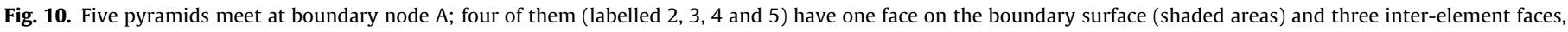
while the remaining (labelled 1 ) one has zero boundary face and four inter-element faces.

\subsubsection{Formulation}

Let us call $\Gamma^{\prime}$ a generic boundary plane and A the boundary node at which the inter element forces and stress tractions are to be ob tained. The local reference system is $\left(x^{\prime}, y^{\prime}, z^{\prime}\right)$, with $z^{\prime}$ normal to the $\Gamma^{\prime}$ plane. The stress tensor in Voigt notation and in local compo nents is $\mathbf{T}^{\prime}$. Let us assume that the $z^{\prime}$ components of the stress ten sor $\mathbf{T}^{\prime}$ (i.e., $\sigma_{z}^{\prime}, \tau_{y z}^{\prime}$ and $\tau_{x z}^{\prime}$ ) are known (boundary conditions). Being $\mathbf{Q}$ the rotation matrix from the local to the global reference system, the stress tensor $\mathbf{T}$ in global components can be written as $\mathbf{T} \quad \mathbf{Q}_{0} \mathbf{T}_{0}^{\prime}+\mathbf{Q}_{1} \mathbf{T}_{1}^{\prime}$ with

$\mathbf{T}_{0}^{\prime} \quad\left[\begin{array}{c}\sigma_{z}^{\prime} \\ \tau_{y z}^{\prime} \\ \tau_{x z}^{\prime}\end{array}\right]$ and $\mathbf{T}_{1}^{\prime} \quad\left[\begin{array}{c}\sigma_{x}^{\prime} \\ \sigma_{y}^{\prime} \\ \tau_{x y}^{\prime}\end{array}\right]$

and $\mathbf{Q}_{0}$ and $\mathbf{Q}_{1}$ the appropriate sub matrices of $\mathbf{Q}$.

With the previous definitions, from this point on the mini mization procedure is very similar to the one for the standard inner nodes presented in Section 4.1.2. That returns the solution:

$\mathbf{r}_{1} \quad \mathbf{K}^{\prime}{ }^{1}\left(\begin{array}{ll}\mathbf{L}^{\prime} \mathbf{f} & \mathbf{t}_{0}\end{array}\right)$

in which

$$
\begin{aligned}
& \mathbf{K}^{\prime} \quad \mathbf{A}^{T} \boldsymbol{\Omega}{ }^{1} \boldsymbol{\Pi}^{\prime} \boldsymbol{\Omega}{ }^{1} \mathbf{A}, \mathbf{L}^{\prime} \quad \mathbf{A}^{T} \boldsymbol{\Omega}{ }^{1} \boldsymbol{\Pi}^{\prime} \boldsymbol{\Omega}{ }^{1} \mathbf{B},
\end{aligned}
$$

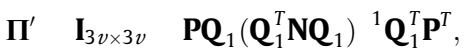

and

$$
\mathbf{t}_{0} \quad \mathbf{A}^{T} \boldsymbol{\Omega}{ }^{1} \boldsymbol{\Pi}^{\prime} \boldsymbol{\Omega}{ }^{1} \mathbf{P}\left[\mathbf{I}_{6 \times 6} \quad \mathbf{Q}_{1}\left(\mathbf{Q}_{1}^{T} \mathbf{N} \mathbf{Q}_{1}\right){ }^{1} \mathbf{Q}_{1}^{T} \mathbf{N}\right] \mathbf{Q}_{0} \mathbf{T}_{0}^{\prime} .
$$

Once the system (28) is solved, one can obtain all the inter element stress vectors from (11), and the remaining nodal inter element forces from (7). The nodal stress tensor $\mathbf{T}$ is again a by product in this case, and can be recovered from:

$$
\mathbf{T}_{1}^{\prime} \quad\left(\mathbf{Q}_{1}^{T} \mathbf{N} \mathbf{Q}_{1}\right){ }^{1}\left(\mathbf{Q}_{1}^{T} \mathbf{c} \quad \mathbf{Q}_{1}^{T} \mathbf{N} \mathbf{Q}_{0} \mathbf{T}_{0}^{\prime}\right),
$$

where $\mathbf{N}$ and $\mathbf{c}$ are the same as defined in (15).

\section{On the contributing area concept}

In this work, the nodal inter element forces $\mathbf{r}$ are related to the inter element stresses at the node $\mathbf{t}$ via a simple diagonal expres sions Eqs. (9) or (10). This implies the intuitive assumption that for a given inter element surface, each inter element nodal force is related to the corresponding inter element stress traction at the same node exclusively, by means of the contributing area $\Omega$. This relation, which is essential for the formulation proposed, seems reasonable and trivial in the case of a constant stress trac tion distribution (Fig. 7), but may deserve some further discussion for other more general situations.

The inter element surface between two 3D finite elements con stitutes a $2 \mathrm{D}$ domain $A$, generally non planar, with a geometry sim ilar to a $3 \mathrm{D}$ shell or interface element, and parametrization in terms of two local coordinates $(r, s)$, i.e.,

$$
\mathbf{x} \quad \mathbf{x}(r, s), \quad \mathbf{x} \quad[x, y, z]^{T} .
$$

The stress traction field transmitted across this surface is denoted as $\mathbf{p}(r, s) \quad\left[p_{x}, p_{y}, p_{z}\right]^{T}(r, s)$, and the displacement field as $\mathbf{u}(r, s)$ $\left[u_{x}, u_{y}, u_{z}\right]^{T}(r, s)$. In the current context of displacement based FEM, the displacement field may be approximated by the nodal values $\mathbf{u}_{e}$ using the shape functions $N^{(i)}(r, s)$, i.e.: 
$\mathbf{u}(r, s) \quad \mathbf{H}(r, s) \mathbf{u}_{e}$

where $\mathbf{H}$ is the traditional matrix array of interpolation or shape functions.

Application of the Principle of Virtual Work (PVW) between the system of applied surface tractions $\mathbf{p}$ and the equivalent nodal forces $\mathbf{r}_{e}$, both subject to a system of virtual nodal displacements $\delta \mathbf{u}_{e}$, and the corresponding virtual displacements at any point on the surface $\delta \mathbf{u}(r, s)$ obtained with (33), leads to:

$\delta \mathbf{u}_{e}^{T} \mathbf{r}_{e} \quad \int_{A} \delta \mathbf{u}^{T}(r, s) \mathbf{p}(r, s) d A \quad \int_{A} \delta \mathbf{u}_{e}^{T} \mathbf{H}^{T}(r, s) \mathbf{p}(r, s) d A$.

Eq. (34) must be satisfied for any values of the virtual nodal dis placement $\delta \mathbf{u}_{e}$. Same as in the classical derivation of nodal forces equivalent to external loads, this finally leads to:

$\mathbf{r}_{e} \quad \int_{A} \mathbf{H}^{T}(r, s) \mathbf{p}(r, s) d A$.

If the above integral is evaluated numerically using an integration rule with NIP points [13], one obtains:

$\mathbf{r}_{e} \sum_{k 1}^{N I P} \mathbf{H}^{T}\left(r_{k}, s_{k}\right) \mathbf{p}\left(r_{k}, s_{k}\right) \Omega_{k}$,

where $\Omega_{k}$ would be the contributing area for integration point $k$, gi ven by:

$\Omega_{k} \quad J_{k} w_{k}$

in which $w_{k}$ is the weight at the $k^{\text {th }}$ integration point, and $J_{k}$ is the Jacobian obtained from the local to global transformations (32).

The former expression (36) may be rearranged in the following matrix form:

$\mathbf{r}_{e} \quad \mathbf{G p}_{e}$,

where

G $\left[\begin{array}{ccc}\Omega_{1} \mathbf{N}_{1}\left(r_{1}, s_{1}\right) & \ldots & \Omega_{N I P} \mathbf{N}_{1}\left(r_{N I P}, s_{N I P}\right) \\ \Omega_{1} \mathbf{N}_{2}\left(r_{1}, s_{1}\right) & \ldots & \Omega_{N I P} \mathbf{N}_{2}\left(r_{N I P}, s_{N I P}\right) \\ \ldots & \ldots & \ldots \\ \Omega_{1} \mathbf{N}_{N N}\left(r_{1}, s_{1}\right) & \ldots & \Omega_{N I P} \mathbf{N}_{N N}\left(r_{N I P}, s_{N I P}\right)\end{array}\right]$

and $N N$ is the number of nodes of the surface element and $\mathbf{p}_{e}$ are the traction stresses at the integration points.
Note that, if the integration points are located exactly at the nodes of the surface element, a double purpose is obtained: (i) ma trix $\mathbf{G}$ becomes diagonal, since the shape functions are always de fined such that their values at corresponding node are 1 and at all other nodal points are zero, and (ii) vector $\mathbf{p}_{e}$ contains the nodal stress tractions.

In this way, as long as the integration points are located at the nodes, Eq. (38) correlates inter element nodal forces to inter ele ment nodal tractions through contributing areas in a decoupled way. This is without making any assumption on the type of distri bution of the stress tractions $\mathbf{p}$ on the surface (such as e.g., constant or linear), i.e., the decoupled one to one relation would be totally general and valid for any distribution of $\mathbf{p}$.

\subsection{Special case for quadratic triangular $\left(\mathbf{T}_{2}\right)$ and serendipity $\left(\mathbf{Q}_{2}^{\prime}\right)$ elements}

Fig. 11 depicts the most usual surface elements used in a context of standard FE codes, both linear and quadratic. On the left side ele ments $\mathbf{I}_{1}$ and $\mathbf{I}_{2}$ represent the only linear and quadratic 1 D surface elements to be used in 2D calculations. For 3D calculations, all ele ments used in practice exhibit either triangular or quadrilateral sides, which are represented in their linear and quadratic version in the central and right columns of Fig. 11, respectively. Note that for quadrilaterals, two versions of quadratic surface elements are possible, the Lagrangian with center face node (denoted as $\mathbf{Q}_{2}$ in the figure) and the serendipity without center face node (denoted as $\mathbf{Q}_{2}^{\prime}$ ). Additionally, we note that all those elements are in general defined isoparametrically, so the edges of the elements in Fig. 11 are straight and the angles for quadrilateral elements are right, just for a matter of simplicity of the drawing.

The study of existing literature leads to the conclusion that inte gration rules with integration points coinciding with the nodes are available for all linear elements $\mathbf{I}_{1}, \mathbf{T}_{1}$ and $\mathbf{Q}_{1}$, with weights equal for all integration points in local coordinates (obviously, the contributing area is also affected by the jacobian of the isoparametric transforma tion which may be different for each point). For the quadratic ele ments, element $\mathbf{I}_{2}$ has the Newton Cotes or Lobatto integration rule with weights 141 ; element $\mathbf{Q}_{2}$ has the corresponding $2 \mathrm{D}$ extension with weights 1416 for corner midside center nodes. The remaining elements $\mathbf{T}_{2}$ and $\mathbf{Q}_{2}^{\prime}$, hoverer, exhibit some peculiarities.
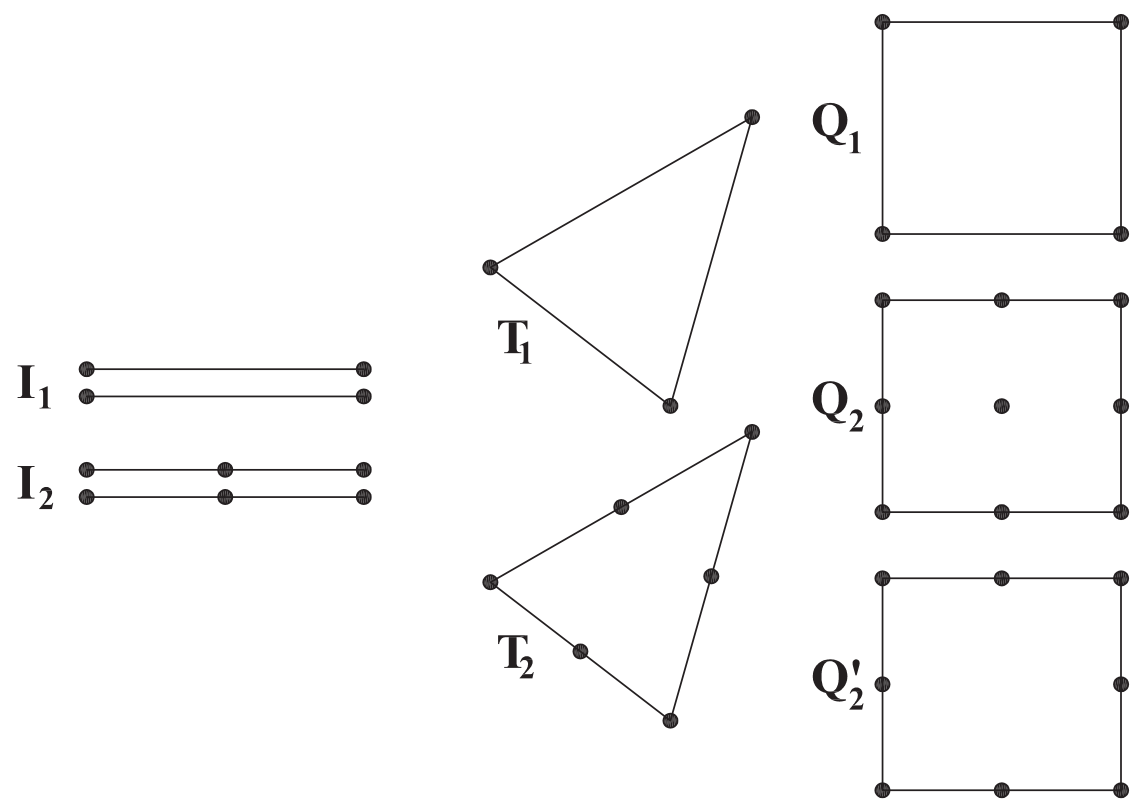

Fig. 11. Finite inter-element surfaces (or interface elements). 
As for element $\mathbf{T}_{2}$, when using a numerical integration rule with integral points coincident with the mid edge and corner nodes [15], the contributing areas turn out to be equal to zero at the corner nodes. This means that the relationship in (9) be tween inter element forces and stresses becomes indeterminate and the proposed method cannot be applied at corner nodes of $\mathbf{T}_{2}$ elements. Nevertheless, matrix $\mathbf{G}$ still gives the values of the contributing areas for the mid edge nodes (equal to $A / 3$, being $A$ the total triangular surface area), and the stress recovery proce dure presented in this paper is still valid for those nodes, as shown by the numerical application presented in Section 6.4.1. The calculation of inter element forces and stresses at corner nodes is still under investigation and it is not presented in this pa per. A possible solution could be found in the extrapolation by lin ear or quadratic functions of the inter element stresses from the mid edge nodes, but this approach is still an on going research topic.
Another special case is element $\mathbf{Q}_{2}^{\prime}$, for which the standard Newton Cotes $3 \times 3$ integration rule contains a central integration point that does not coincide with any node, leading therefore to the loss of the diagonal character of matrix $\mathbf{G}$. In this particular situa tion, Eq. (38) becomes:

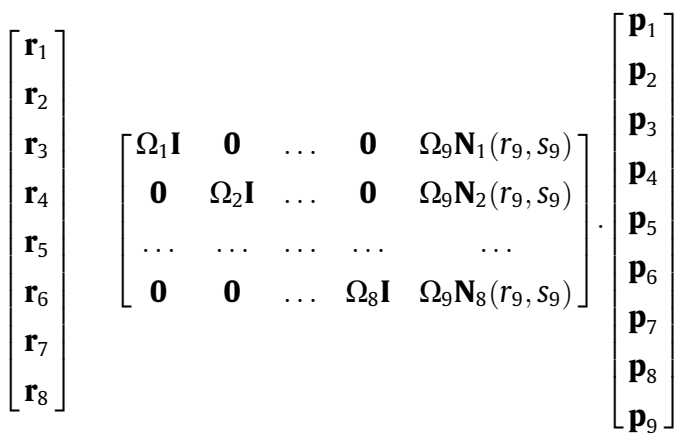

(a)
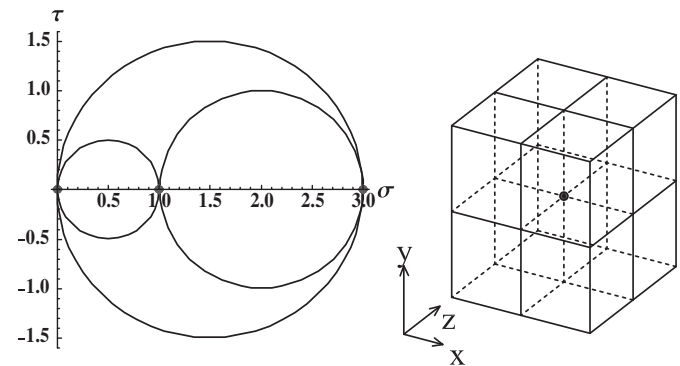

(b)
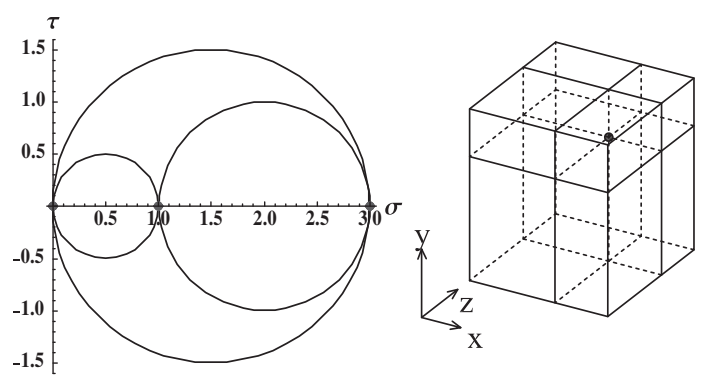

(c)
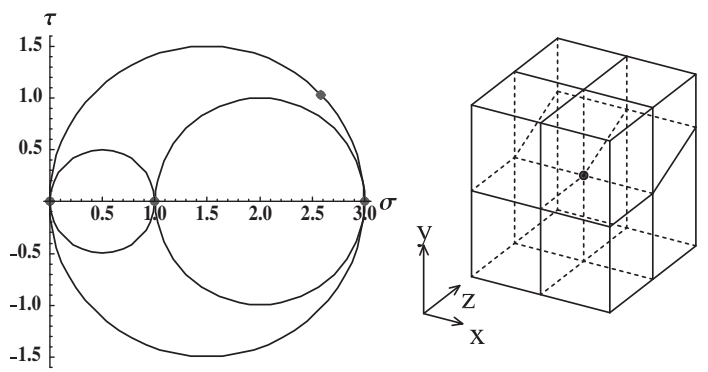

(d)
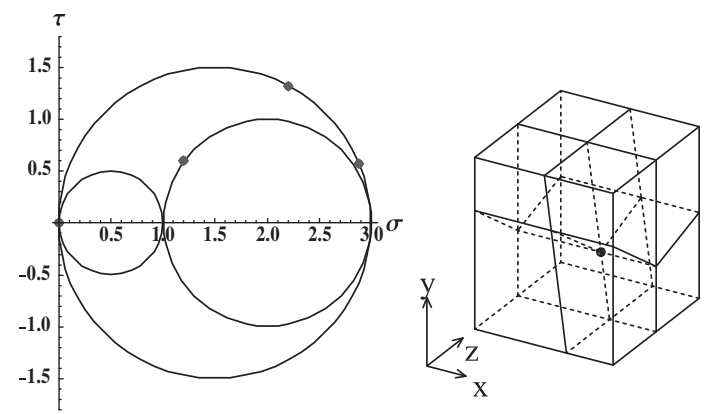
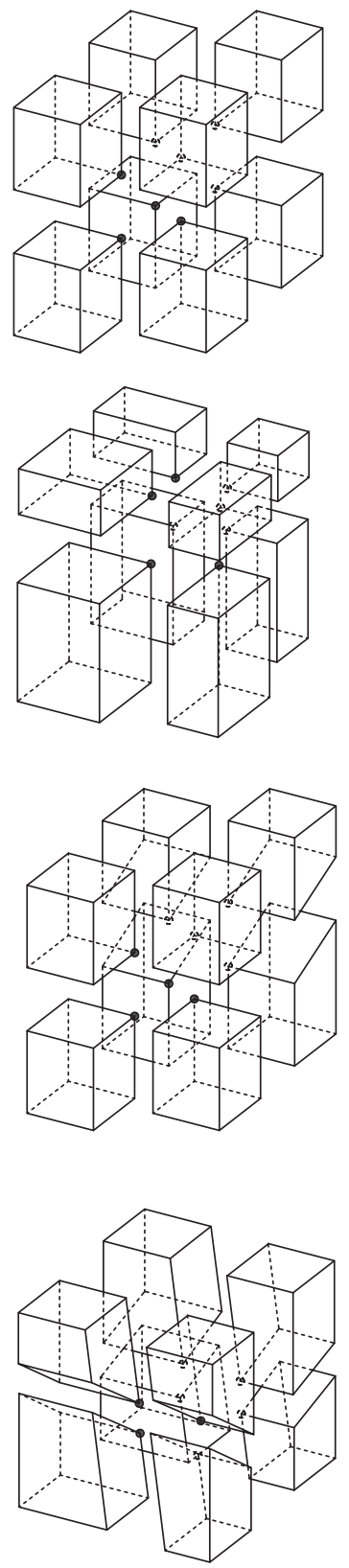

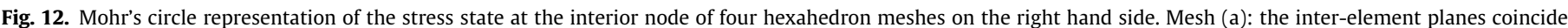

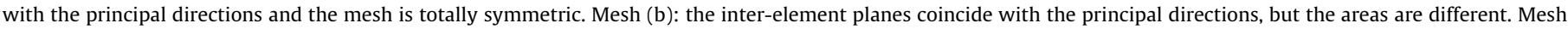
(c): inter-element planes coincide with the principal directions,except one. Mesh (d): only one of the planes coincides with the principal directions. 

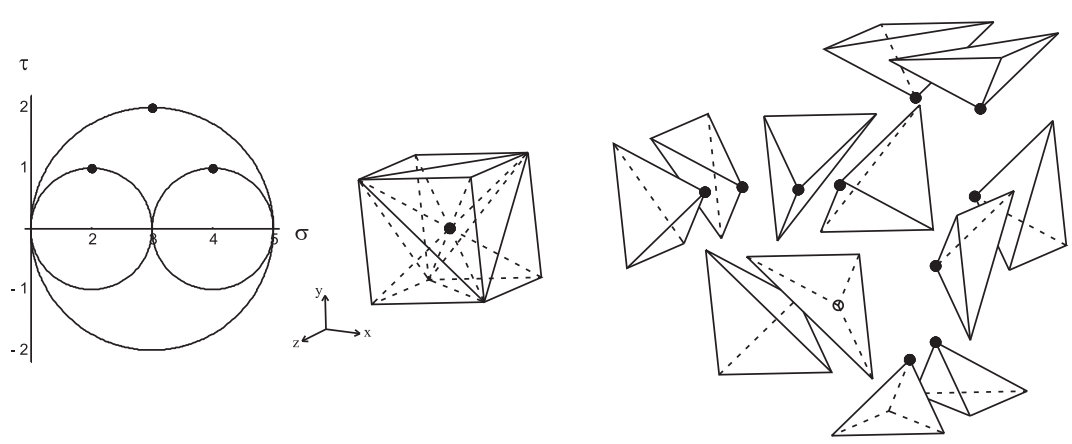

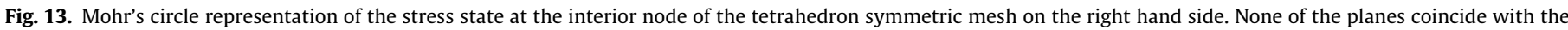
principal directions, but all the stress vectors are plane.
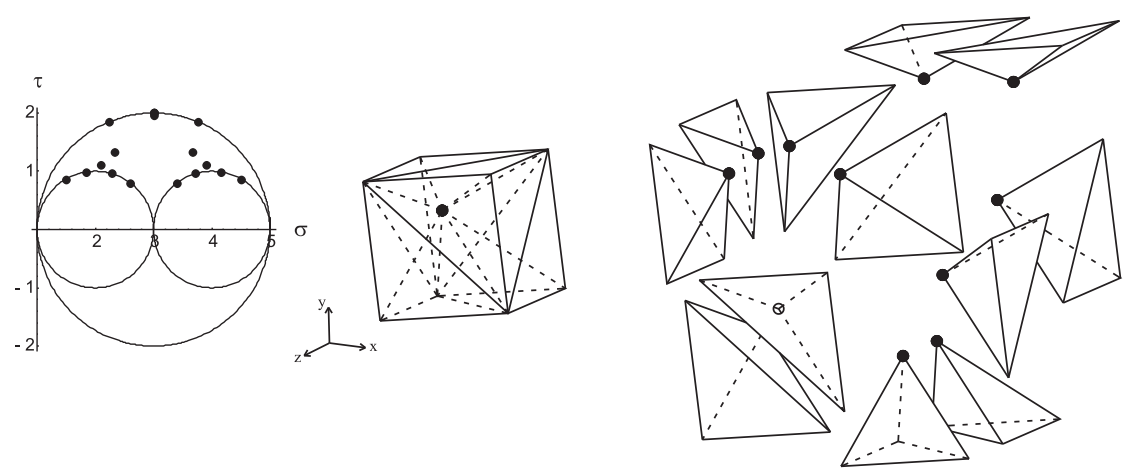

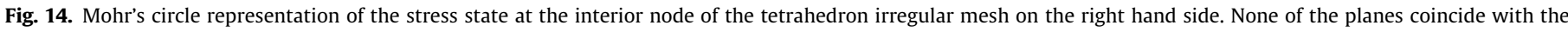
principal directions; some of the stress vectors are plane and some are not.

Table 1

Exact solution and numerical error of the recovered stress tensor components for the case of gravity load of meshes in Fig. 12.

\begin{tabular}{|c|c|c|c|c|c|c|c|c|c|}
\hline \multirow[t]{2}{*}{ Mesh } & \multirow[t]{2}{*}{$\begin{array}{l}y^{\prime} \\
(\mathrm{m})\end{array}$} & \multicolumn{2}{|c|}{ Exact solution } & \multicolumn{3}{|c|}{$\begin{array}{l}\text { Error in linear } \\
\text { analysis }\end{array}$} & \multicolumn{3}{|c|}{$\begin{array}{l}\text { Error in } \\
\text { quadratic } \\
\text { analysis }\end{array}$} \\
\hline & & $\begin{array}{l}\sigma_{y} \\
(\mathrm{~Pa})\end{array}$ & $\begin{array}{l}\sigma_{z} \\
(\mathrm{~Pa})\end{array}$ & $\begin{array}{l}\sigma_{y} \\
(\%)\end{array}$ & $\begin{array}{l}\sigma_{z} \\
(\%)\end{array}$ & $\begin{array}{l}\sigma_{x} \\
(\%)\end{array}$ & $\begin{array}{l}\sigma_{y} \\
(\%)\end{array}$ & $\begin{array}{l}\sigma_{z} \\
(\%)\end{array}$ & $\sigma_{x}$ \\
\hline (a) & 0.5 & 9 & 4.4 & 0 & 0 & 0 & 0 & 0 & \\
\hline (b) & 0.3 & 5.4 & 2.6 & 0.3 & 52.0 & 49.8 & 0 & 0 & \\
\hline (c) & 0.5 & 9 & 4.4 & 4.3 & 4.5 & 10.6 & 0 & 0 & \\
\hline (d) & 0.6 & 10.8 & 5.3 & 8.6 & 12.8 & 2.8 & 0 & 0 & \\
\hline
\end{tabular}

in which 1, 2, 3, 4 indicate corner nodes, 5, 6, 7, 8 mid edge nodes and 9 the integration point at the center of the face. For the general isoparametrical $\mathbf{Q}_{2}^{\prime}$ element of unit area of Fig. 11, (40) turns into:

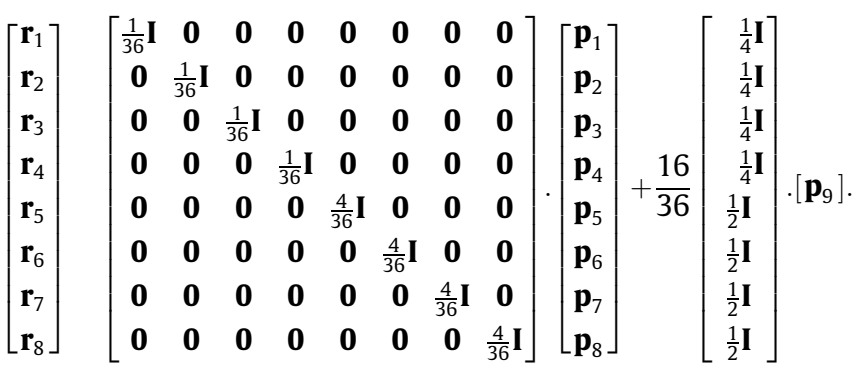

Under the assumption of constant stress distribution (i.e., $\mathbf{p}(r, s) \quad \mathbf{p}$ constant), the previous equation becomes:

$\left[\begin{array}{l}\mathbf{r}_{1} \\ \mathbf{r}_{2} \\ \mathbf{r}_{3} \\ \mathbf{r}_{4} \\ \mathbf{r}_{5} \\ \mathbf{r}_{6} \\ \mathbf{r}_{7} \\ \mathbf{r}_{8}\end{array}\right] \quad\left[\begin{array}{c}\frac{1}{12} \mathbf{I} \\ \frac{1}{12} \mathbf{I} \\ \frac{1}{12} \mathbf{I} \\ \frac{1}{12} \mathbf{I} \\ \frac{1}{3} \mathbf{I} \\ \frac{1}{3} \mathbf{I} \\ \frac{1}{3} \mathbf{I} \\ \frac{1}{3} \mathbf{I}\end{array}\right] \cdot[\mathbf{p}]$.

With Eq. (42), the de coupled relationship between nodal inter ele ment forces and stresses is restored, but with the limitation of being strictly satisfied only for constant stress states on the inter element surface. Furthermore, the tributary areas associated to the corner nodes appear negative. An example for the calculation of inter ele ment tractions at the mid edge nodes using a contributing area equal to $A / 3$ is presented in Section 6.4.2. Even in this case, the indeterminacy of the inter element tractions at corner nodes is still under investigation and a solution is not proposed in this paper.

\section{Examples}

In this section, some numerical applications are presented to validate the accuracy of the proposed method. In all the following examples, the material is assumed linear isotropic elastic loading is sufficiently simple so that the exact theoretical stress state at the node and inter element stress tractions are known.

It is important to recall here that the main purpose of this meth od is to evaluate inter element stress tractions transmitted across inter element planes, while a stress tensor at the node itself is ob tained as a least square by product of the calculation. One key fea ture of the method is that equilibrium is strictly enforced between 
all nodal forces (continuous element nodal forces and inter ele ment forces). Enforcement of this condition may cause the corre sponding stress tractions on the inter element planes (which are equal to the corresponding inter element forces divided each of them by the corresponding contributing area) not to be all of them exactly equal to the projections of a unique nodal stress tensor onto those planes, but only the best approach by least squares. This may happen when the element order is lower than the order re quired to reproduce the exact solution (i.e., linear elements with non uniform load). In those cases, also the nodal stress tensor (by product of the minimization) may turn out not exactly equal to the theoretical one. This case is illustrated in some of the exam ples below, and also it is shown how the differences with the the oretical solution vanish as the mesh is refined.

\subsection{Inter element tractions at interior corner nodes of linear hexahedra and tetrahedra under uniform stress state}

\subsubsection{Linear hexahedra}

In this first set of examples, four different meshes of a cubical domain are subject to a uniform stress state with principal axes $x$, $y, z$. The external loads applied correspond to a uniform stress state of $\sigma_{x} \quad 1 \mathrm{~Pa}, \sigma_{y} 3 \mathrm{~Pa}, \sigma_{z} \quad 0 \mathrm{~Pa}$ and $\tau_{x y} \tau_{y z} \quad \tau_{x z} \quad 0$ The discretization is made with eight linear hexahedral elements (Fig. 12) introducing progressively higher degree of irregularity in the mesh.

In the first case (mesh a in Fig. 12) the subdivision of the cube is made along planes parallel to the coordinate axes with uniform element size (i.e., constant contributing areas); in the second case (mesh b) the contributing areas of the elements concurrent to the internal corner node are different; in the third and fourth cases (mesh $\mathrm{c}$ and d) some of the mesh subdivisions are not aligned with the coordinate axes. In all cases, the exact stress tractions at the in ter element planes around the interior corner node are obtained, as shown on the left column of Fig. 12. Note that due to the orien tation of the inter element planes, tractions in meshes a and $b$ have no shear component, and tractions in meshes $\mathrm{c}$ and $\mathrm{d}$ exhibit shear components as expected.

The solution in (11) gives the iter element tractions in global coordinates. The representation in the Mohr's plane implies the transformation from global $\left[t_{x}, t_{y}, t_{z}\right]$ to local $\left[\sigma, \tau_{1}, \tau_{2}\right]$ coordinates. For a matter of simplicity in this and the following examples the shear components $\left[\tau_{1}, \tau_{2}\right]$ are represented in their absolute values. This does not mean that the method is not able to recover the trac tions with their correct sign.

\subsubsection{Linear tetrahedra}

In this second set of examples a cubical domain is discretized with linear tetrahedral elements, first using a regular symmetric mesh shown in Fig. 13, and then using an irregular mesh (Fig. 14). The external load applied corresponds to a uniform stress state of

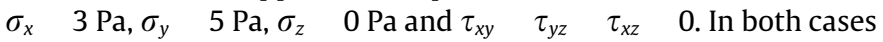
the correct nodal stress tractions are recovered, as well as the no dal stress tensor as shown on the left of Figs. 13 and 14. Note that in the regular case (Fig. 13) the resulting stress tractions lie on the maximum shear point of each of the three circles of the 3D Mohr diagram; in contrast, in the irregular mesh (Fig. 14) some of the in ter element planes do not correspond to such specific orientations and therefore the corresponding points on the Mohr diagram are spread over the three circles and the region between them.

\subsection{Inter element tractions at interior corner nodes of linear and quadratic hexahedra under non uniform stress state}

The aim of these applications is to show that the accuracy of the recovered tractions depends on the accuracy of the pre processed nodal forces obtained by the standard FE analysis. The meshes are the same already used in the previous section (represented in the central column of Fig. 12), which now are subject to the gravity load (along $y$ axis in the figure) with no deformation allowed along the horizontal directions (i.e., axes $x, z$ in the figure). Linear first and quadratic hexahedral elements then are used for the four (b)

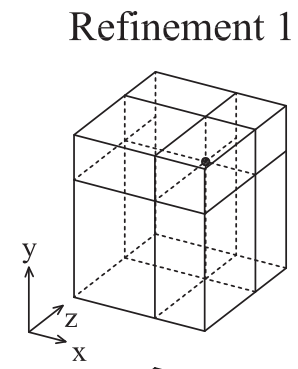

(c)

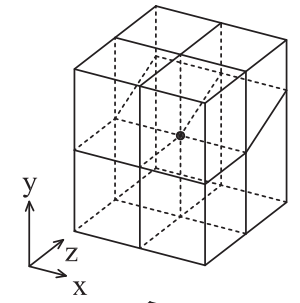

(d)

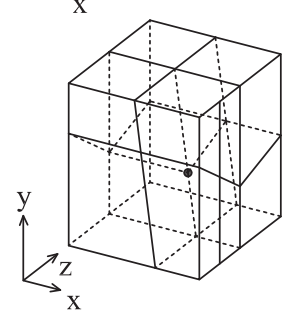

Refinement 2
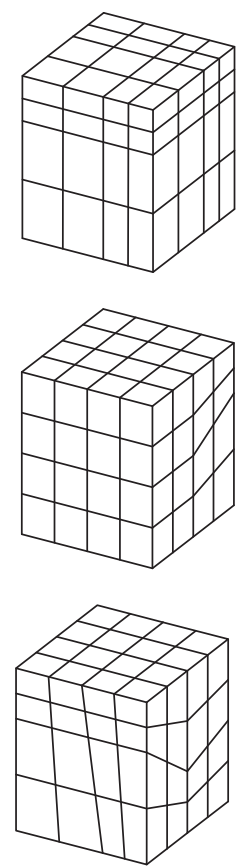
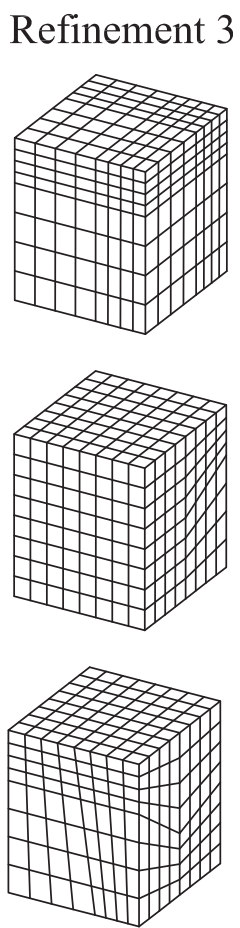

Fig. 15. Refinements of meshes (c) and (d) of Fig. 12. 

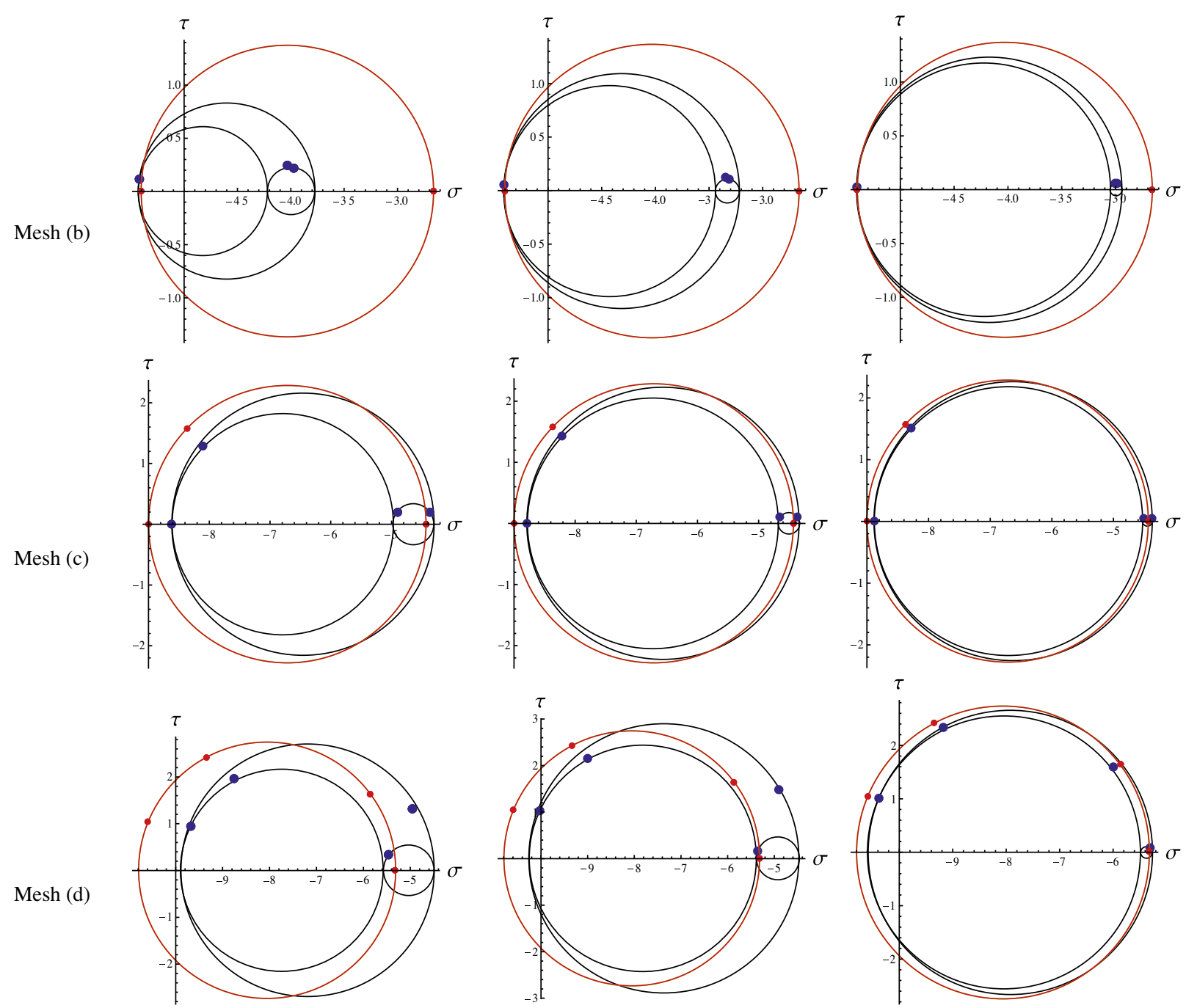

Fig. 16. In blue: the stress tractions and stress tensors at the interior node of the linear meshes shown in Fig. 15. In red: the theoretical solution.
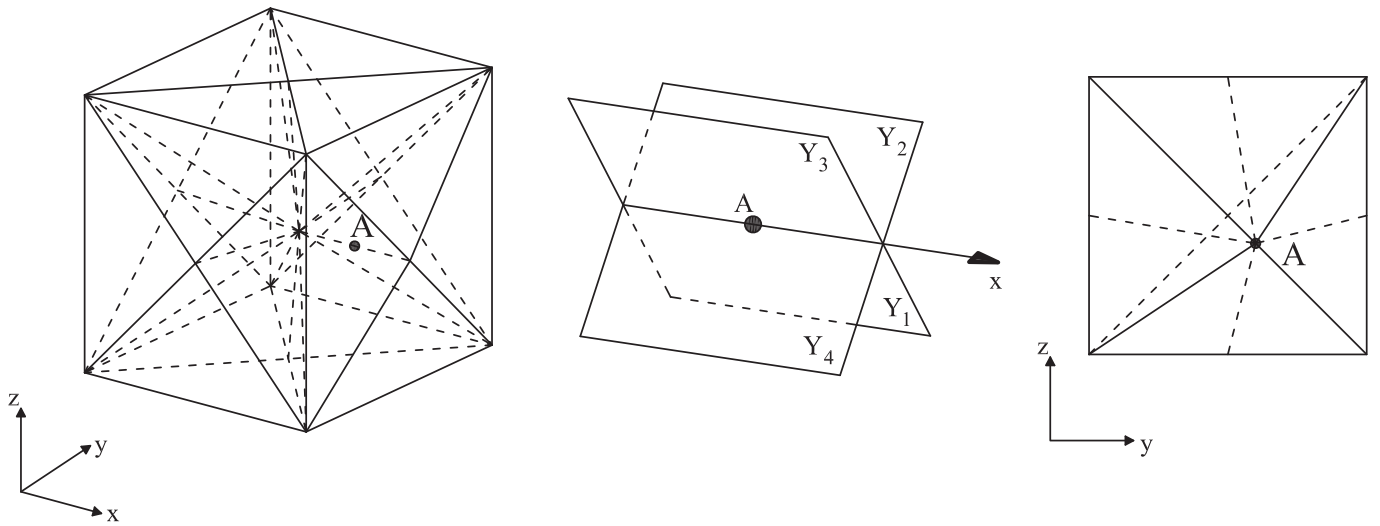

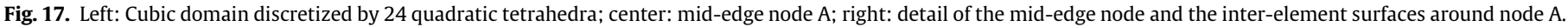

meshes. As known, linear elements and coarse refinements per form badly when a non uniform stress state is applied. On the other side, in case of gravity load, quadratic elements perform well even with coarse meshes. This condition applies to the results of the proposed method, as shown in the following example.
For all the examples, it has been assumed that the specific density $\rho$ is equal to $1.8 \mathrm{~kg} / \mathrm{m}^{3}$, the Poisson coefficient $v \quad 0.33$ and the gravity acceleration $\|\mathbf{g}\| \quad g \quad 10 \mathrm{~m} / \mathrm{s}^{2}$. At each point of the continuum solid, the theoretical stress tensor is given by: 
Table 2

Error (in \%) of the recovered stress tractions of Fig. 17.

\begin{tabular}{lllll}
\hline & Plane $Y_{1}$ & Plane $Y_{2}$ & Plane $Y_{3}$ & Plane $Y_{4}$ \\
\hline$t_{x}$ & 0 & 0 & 0 & 0 \\
$t_{y}$ & 0.13 & 0.16 & 0.13 & 0.11 \\
$t_{z}$ & 0.26 & 0.45 & 0.26 & 0.33 \\
\hline
\end{tabular}

$\mathbf{T}\left[\begin{array}{ccc}\sigma_{x} & \tau_{x y} & \tau_{x z} \\ \tau_{x y} & \sigma_{y} & \tau_{y z} \\ \tau_{x z} & \tau_{y z} & \sigma_{z}\end{array}\right] \quad\left[\begin{array}{ccc}K_{0} \sigma_{y} & 0 & 0 \\ 0 & \sigma_{y} & 0 \\ 0 & 0 & K_{0} \sigma_{y}\end{array}\right]$ with $K_{0} \frac{v}{1} v, \sigma_{y} \quad \rho g y^{\prime}$

being $y^{\prime}$ the distance from the top surface.

Note that from the physical viewpoint, $\sigma_{y}$ will be different for each mesh depending on the vertical position of the interior node under study. Also, with the boundary conditions used, $\sigma_{x}$ should be the same as $\sigma_{z}$. Table 1 summarizes, for each of the four meshes, the vertical location of the central node ( $y^{\prime}$ coordinate), the analyt ical exact solution of the vertical and horizontal stresses, as well as the values of the error of the nodal stress tensor components ob tained using the proposed method, for both linear and quadratic elements.

It is evident that the linear elements give inaccurate results while the quadratic elements catch the theoretical stress state, as shown in the Table 1 (except for the totally symmetric mesh (case a) that shows no difference between the linear and the quadratic meshes).

It has to be noted, as previously mentioned, that the linear analysis presented here is a classical well known case in which the interpolation order of the elements (linear) is lower than that required to represent the displacement field (quadratic). Therefore, the overall results of the FE calculation using linear elements could be expected to be clearly inaccurate in terms of stresses, especially if only a few elements are used in the discretization, which is exactly the case in the small academic examples presented (with only two elements per direction). This error would be reduced
Table 3

Error (in \%) of the non-zero recovered stress tractions of Fig. 18.

\begin{tabular}{llll}
\hline Plane & Non-zero component & Analytical exact solution & Error (\%) \\
\hline 1 & $t_{x}$ & -29.55 & 0 \\
2 & $t_{y}$ & -60.0 & 0.016 \\
3 & $t_{x}$ & -29.55 & 0 \\
4 & $t_{y}$ & -60.0 & 0.016 \\
\hline
\end{tabular}

progressively as the linear mesh is refined, as it is shown in the fol lowing section.

\subsection{Refinement study}

Similarly to the previous examples, the following applications show that the accuracy of the proposed method strongly depends on the level of accuracy of the pre processed FE analysis. Geometry (b), (c) and (d) of Fig. 12 are re proposed in Fig. 15 with different refinements using linear hexahedral elements under gravity load. The stress tractions at the interior node (black point shown along the left column of Fig. 15) are plotted in the Mohr's plane in Fig. 16. The results are shown in blue, together with the theoretical solutions (43) in red. It is evident that refined linear meshes per form better than coarse meshes, with a clear trend of convergence towards the correct solution as long as the mesh is refined.

\subsection{Inter element tractions at mid edge nodes}

\subsubsection{Quadratic tetrahedra}

The cubic domain shown on the left side of Fig. 17 is discretized using 24 quadratic tetrahedral elements. The nodal inter element stresses at the mid edge node $A$ of the four elements around $A$ are recovered, as well as the five components of the stress tensor in $A$, when the sample is subjected to a gravity load case similar to the one in Section 6.2 and 6.3.

Note that all the normal vectors of the inter element surfaces in A are contained in the $y \quad z$ plane, i.e., the direction of the edge to which node A belongs (indicated as $\mathbf{m}$ in Fig. 8) coincides with $\mathbf{x}$.
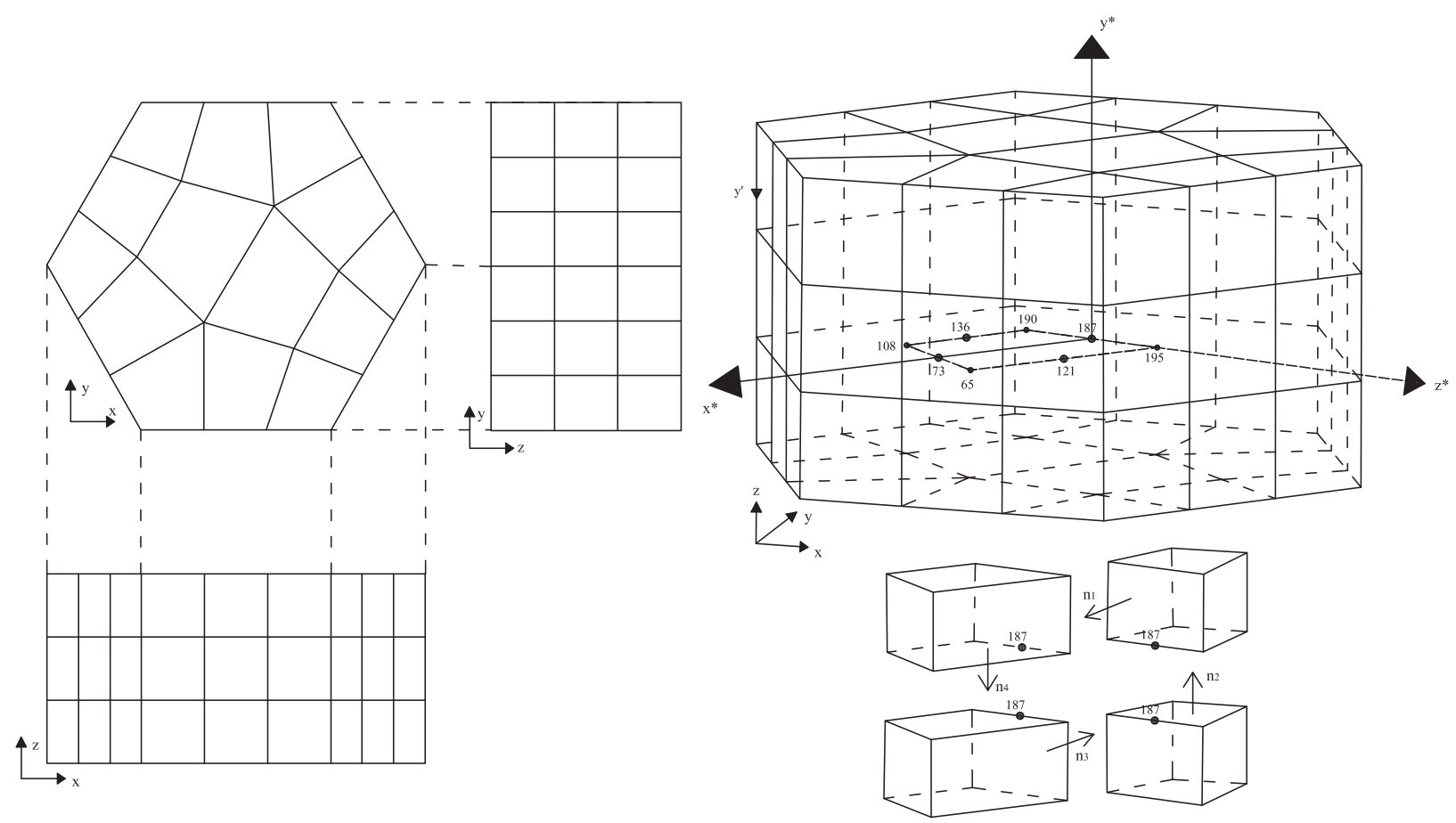

Fig. 18. Example of calculation of inter-element forces around a mid-edge node of a hexahedron (serendipity-type) element. 

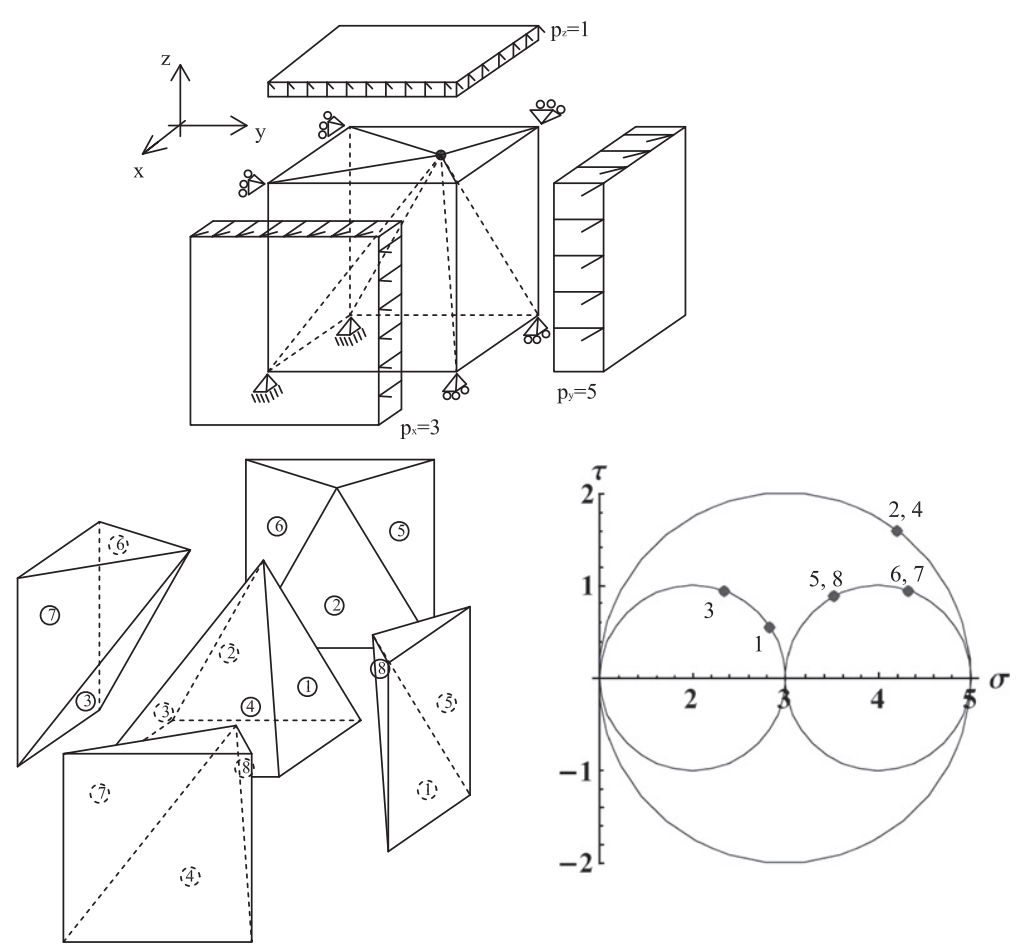

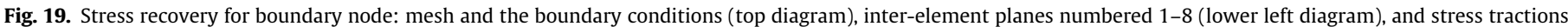
represented by dots on the Mohr plane with the Mohr circles on the right (lower right diagram).

Hence, the x component of all normal vectors $\mathbf{n}_{i}$ to the planes $Y_{1}$ ( $i \quad 1,4)$ is equal to zero. Also for this example, the exact solution is taken from (43). Using the proposed method, the error of the recovered inter element stress tractions (in $\mathrm{Pa}$ ) is presented in $\mathrm{Ta}$ ble 2. The method shows accurate results also in this case.

Of the stress tensor at point A, as a by product of the proposed formulation, only five components can be recovered in this case while the $\sigma_{x}$ component of the stress tensor $\mathbf{T}$ at this node remains undetermined.

\subsubsection{Quadratic hexahedra}

The mesh is Fig. 18 is made of 14 serendipity elements. For these elements, in order to maintain the relationship in (9) and to have a diagonal matrix $\boldsymbol{\Omega}$ the assumption that the stress field on the surface is constant is necessary, as explained in Section 5.1. The mesh is subject to a gravity load along $\mathrm{z}$, with no deforma tion allowed along the horizontal directions (i.e., axes $x$ and $y$ in Fig. 18). The inter element tractions around the mid edge node 187 have been calculated. The analytical solution of the stress trac tions $\mathbf{t}^{(i)} \quad\left[t_{x}, t_{y}, t_{z}\right]^{(i)}$ on the four planes around point 187 exhibit all zero values except for the components perpendicular to the plane. Table 3 presents the non zero analytical solution and the er ror of the relative recovered tractions obtained with the proposed minimization.

It is interesting to note that the method gives accurate results also for the inter element surfaces 1 and 3 (indicated at the bot tom right side of Fig. 18) upon which the stress field is not con stant, contrary to the assumptions used in this method with $Q^{\prime} 2$ type elements.

\subsection{Inter element tractions at boundary nodes}

In this example, a $1 \mathrm{~m} \times 1 \mathrm{~m} \times 1 \mathrm{~m}$ cubical domain is subject to a uniform stress state of 3, 5 and 8 Pa respectively along $x, y$ and $z$. The cube is discretized with five linear pyramid elements of quadrangular base, arranged as shown in the upper diagram in Fig. 19, so that the tips of all them are connected at the node lo cated at the center of the upper face of the cube. The formulation developed in Section 6 is applied to this boundary node.

Eight inter element surfaces numbered 18 in the lower left diagram of Fig. 19 are connected to the boundary node in analysis. Due to the load configuration, the principal stresses are in this case equal to the values of the applied loads. For symmetry with respect to the $y$ axis, the stress tractions on planes 5 and 8 coincide, as they also do for planes 2 and 4, and for planes 6 and 7. The calculated stress tractions and $\mathbf{T}$ tensor are presented on the lower right side of Fig. 19. They coincide with the theoretical exact solution.

\section{Concluding remarks}

The formulation proposed by the authors in [1] for the evalua tion of inter element forces and stress tensor at the nodes in 2D FE meshes has been extended in 3D for the recovery of inter ele ment stress tractions alone. These quantities may be evaluated at the nodes of a standard 3D FE displacement mesh as a post pro cessing operation involving the solution of a local linear algebraic system of limited size for each node (in the order of 1218 equa tions in the examples presented). The procedure has been devel oped for corner and mid edge nodes. For the mid edge ones, the proposed method works with any type of element. For corner nodes the method works with any linear element and with the 27 node (quadratic) hexahedron element. For the 13 and 14 node pyramid, 10 node tetrahedron and 20 node hexahedron the calcu lation of the inter element tractions at corner nodes is still under investigation.

In the academic examples provided, the inter element tractions obtained in this way turn out very accurate in most cases, except perhaps in the cubical specimen discretized unsymmetrically with two linear elements per direction and subject to gravity loads, as discussed in the corresponding section. In those examples, the 
accuracy of the method depends on the correctness of the pre pro cessed element nodal forces. Therefore the method do not show any superconvergent property [16 18]. However, when the nodal forces are correctly calculated through the FE analysis, the 3D tech nique presented in this paper may be confirmed as an accurate yet inexpensive way to evaluate the stress tractions transmitted across inter element surfaces throughout the mesh, which are required in studies of crack nucleation along mesh lines.

\section{Acknowledgements}

The second author acknowledges research grants BIA2009 10491 and BIA2012 36898 from MEC as well as grant 2009SGR 180 from Generalitat de Catalunya. The MEC projects included FEDER funds from the European Union which are gratefully acknowledged, as it is also the qualification of the research group as "Unidad Asociada" of CSIC Madrid. The third author wishes to thank the Italian Ministry of Research (MIUR) for the financial sup port received.

\section{References}

[1] D. Ciancio, I. Carol, M. Cuomo, On inter-element forces in the FEMdisplacement formulation, and implications for stress recovery, International Journal of Numerical Method in Engineering 66 (2006) 502-528.

[2] A. Hillerborg, M. Modeer, P.E. Petersson, Analysis of crack formation and crack growth in concrete by means of fracture mechanics and finite elements, Cement Concrete 6 (1976) 773-782.

[3] D. Ciancio, I. Carol, M. Cuomo, Crack opening conditions at 'corner nodes' in FE analysis with cracking along mesh lines, Engineering Fracture Mechanics 74 (2007) 1963-1982.

[4] J.G. Rots, Computational Modeling of Concrete, Ph.D. thesis, TU Delft, 1988.

[5] I. Carol, C.M. Lopez, O. Roa, Micromechanical analysis of quasi-brittle materials using fracture-based interface elements, International Journal for Numerical Methods in Engineering 52 (1/2) (2001) 193-215. [6] A. Caballero, I. Carol, C.M. Lopez, A meso-level approach to the 3D numerical
analysis of cracking and fracture of concrete materials, Fatigue \& Fracture of Engineering Materials \& Structures 29 (12) (2006) 979-991.

[7] J.G. Rots, J.C.J. Schellekens, Interface elements in concrete mechanics, in: N. Bićanić, H. Mang (Eds.), Computer-Aided Analysis and Design of Concrete, vol 2, Pineridge Press, Zell-am-See, Austria, 1990, pp. 909-918.

[8] D. Garolera, C.M. Lopez, I. Carol, P. Papanastasiou, Micromechanical analysis of the rock sanding problem, Journal of the Mechanical Behaviour of Materials, 2005;16(1-2):45-53.

[9] A.R. Ingraffea, V.E. Saouma, Numerical modelling of dicrete crack propagation in reinforced and plain concrete, in: G.C. Sih, A. DiTomasso (Eds.), Fracture Mechanics of Concrete, Martinus Nijhiff: Dordrecht, The Netherlands, 1985, pp. 171-225.

[10] T.N. Bittencourt, A.R. Ingraffea, J. Llorca, Simulation of arbitrary, cohesive crack propagation, in: Z.P. Bažant (Ed.), Fracture Mechanics of Concrete Structures (FRAMCOS1), Elsevier: Breckenridgr, Colorado, 1992, pp. 171-225.

[11] G.T. Camacho, M. Ortiz, Computational modelling of impact damage in brittle materials, International Journal of Solids and Structures 33 (20-22) (1996) 2899-2938.

[12] M. Ortiz, A. Pandolfi, Finite-deformation irreversible cohesive elements for the three-dimensional crack-propagation analysis, International Journal for Numerical Methods in Engineering 44 (9) (1999) 1267-1282.

[13] A. Gens, I. Carol, E. Alonso, An interface element formulation for the analysis of soil-reinforcement interaction, Computers and Geotechnics 7 (1988) 133-151.

[14] T. Belytschko, Wing Kam Liu, B. Moran, Nonlinear Finite Elements for Continua and Structures, Wiley, 2000.

[15] P. Silvester, Symmetric Quadratire Folrmulae for Simplexes, Mathematics of Computation 24109 (1970) 95-100.

[16] O.C. Zienkiewicz, J.Z. Zhu, The superconvergent patch recovery and a posteriori error estimates. Part 1: the recovery technique, International Journal for Numerical Methods in Engineering 33 (1992) 1331-1364.

[17] A.R. Khoei, S.A. Gharehbaghi, The superconvergent patch recovery technique and data transfer operators in 3D plasticity problems, Finite Elements in Analysis and Design 43 (2007) 630-648.

[18] J.J. Rodenas, M. Tur, F.J. Fuenmayor, A. Vercher, Improvement of the superconvergent patch recovery technique by the use of constraint equations: The SPR-C technique, International Journal for Numerical Methods in Engineering 70 (2007) 705-727.

[19] L. Rosati, A novel approach to the solution of the tensor equation $A X+X A=H$, International Journal of Solids and Structures 37 (2000) 3457-3477. 